\title{
INDISTINGUISHABILITY OF PERCOLATION CLUSTERS
}

\author{
By Russell Lyons ${ }^{1}$ AND Oded Schramm ${ }^{2}$ \\ Indiana University and Weizmann Institute of Science
}

\begin{abstract}
We show that when percolation produces infinitely many infinite clusters on a Cayley graph, one cannot distinguish the clusters from each other by any invariantly defined property. This implies that uniqueness of the infinite cluster is equivalent to nondecay of connectivity (a.k.a. long-range order). We then derive applications concerning uniqueness in Kazhdan groups and in wreath products and inequalities for $p_{u}$.
\end{abstract}

1. Introduction. Grimmett and Newman (1990) showed that if $T$ is a regular tree of sufficiently high degree, then there are $p \in(0,1)$ such that Bernoulli $(p)$ percolation on $T \times \mathbb{Z}$ has infinitely many infinite components a.s. Benjamini and Schramm (1996) conjectured that the same is true for any Cayley graph of any finitely generated nonamenable group. (A finitely generated group $\Gamma$ is nonamenable iff its Cayley graph satisfies $\inf _{K}|\partial K| /|K|>0$, where $K$ runs over the finite nonempty vertex sets. See Section 2 for all other definitions.) This conjecture has been verified for planar Cayley graphs of high genus by Lalley (1998) and for all planar lattices in the hyperbolic plane by Benjamini and Schramm (1998).

The present paper is concerned with the percolation phase where there are multiple infinite clusters. We show that under quite general assumptions, the infinite clusters are indistinguishable from each other by any invariantly defined property.

Let $G$ be a Cayley graph of a finitely generated group (more generally, $G$ can be a transitive unimodular graph).

THEOREM 1.1. Consider Bernoulli bond percolation on $G$ with some survival parameter $p \in(0,1)$, and let $\mathscr{A}$ be a Borel measurable set of subgraphs of $G$. Assume that $\mathscr{A}$ is invariant under the automorphism group of $G$. Then either a.s. all infinite percolation components are in $\mathscr{A}$, or a.s. they are all outside of $\mathscr{A}$.

For example, $\mathscr{A}$ might be the collection of all transient subgraphs of $G$, or the collection of all subgraphs that have a given asymptotic rate of growth or the collection of all subgraphs that have no vertex of degree 5 .

\footnotetext{
Received December 1998.

${ }^{1}$ Supported in part by Varon Visiting Professorship at the Weizmann Institute of Science and NSF Grant DMS-98-02663.

${ }^{2}$ Supported in part by the Sam and Ayala Zacks Professorial Chair.

AMS 1991 subject classifications. Primary 82B43, 60B99; secondary 60K35, 60D05.

Key words and phrases. Finite energy, Cayley graph, group, Kazhdan, wreath product, uniqueness, connectivity, transitive, nonamenable.
} 
If $\mathscr{A}$ is the collection of all transient subgraphs of $G$, then this shows that almost surely, either all infinite clusters of $\omega$ are transient (meaning that simple random walk on them is transient), or all clusters are recurrent. In fact, as shown in Proposition 3.11, if $G$ is nonamenable, then a.s. all infinite clusters are transient if Bernoulli percolation produces more than one infinite component. In Benjamini, Lyons and Schramm (1999), it is shown that the same is true if Bernoulli percolation produces a single infinite component.

Theorem 1.1 is a particular case of Theorem 3.3 below, which applies also to some non-Bernoulli percolation processes and to more general $\mathscr{A}$.

A collection of subgraphs $\mathscr{A}$ in $G$ is increasing if whenever $H \in \mathscr{A}$ and $H \subset H^{\prime} \subset G$, we have $H^{\prime} \in \mathscr{A}$. Häggström and Peres (1999) have proved that for increasing $\mathscr{A}$, Theorem 1.1 holds, except possibly for a single value of $p \in(0,1)$. They have also proved the following theorem.

THeOREM 1.2 (Uniqueness monotonicity). Let $p_{1}<p_{2}$ and $\mathbf{P}_{i}(i=1,2)$ be the corresponding Bernoulli $\left(p_{i}\right)$ bond percolation processes on $G$. If there is a unique infinite cluster $\mathbf{P}_{1}$-a.s., then there is a unique infinite cluster $\mathbf{P}_{2}$-a.s. Furthermore, in the standard coupling of Bernoulli percolation processes, if there exists an infinite cluster $\mathbf{P}_{1}$-a.s., then a.s. every infinite $p_{2}$-cluster contains an infinite $p_{1}$-cluster.

Here, we refer to the standard coupling of $\operatorname{Bernoulli}(p)$ percolation for all $p$, where each edge $e \in \mathrm{E}$ is assigned an independent uniform $[0,1]$ random variable $U(e)$ and the edges where $U(e) \leq p$ are retained for $\operatorname{Bernoulli}(p)$ percolation.

The first part of this theorem partially answers a question of Benjamini and Schramm (1996); the full answer, removing the assumption of unimodularity, has been provided by Schonmann (1999a). It follows from Theorem 1.2 that the set of $p$ such that Bernoulli $(p)$ bond percolation on $G$ has more than one infinite cluster a.s. is an interval, called the nonuniqueness phase. It is well known that in the nonuniqueness phase, the number of infinite clusters is a.s. infinite [see Newman and Schulman (1981)]. The interval of $p$ such that Bernoulli $(p)$ percolation on $G$ produces a single infinite cluster a.s. is called the uniqueness phase. The infimum of the $p$ in the uniqueness phase is denoted $p_{u}=p_{u}(G)$.

To illustrate the usefulness of Theorem 1.1, we now show that it implies Theorem 1.2.

Proof of Theorem 1.2. Suppose that there exists an infinite cluster $\mathbf{P}_{1}$-a.s. Let $\omega$ be the open subgraph of the $\mathbf{P}_{2}$ process and let $\eta$ be an independent Bernoulli $\left(p_{1} / p_{2}\right)$ percolation process. Thus, $\omega \cap \eta$ has the law of $\mathbf{P}_{1}$ and, in fact, $(\omega \cap \eta, \omega)$ has the same law as the standard coupling of $\mathbf{P}_{1}$ and $\mathbf{P}_{2}$. By assumption, $\omega \cap \eta$ has an infinite cluster a.s. Thus, for some cluster $C$ of $\omega$, we have $C \cap \eta$ is infinite with positive probability, hence, by Kolmogorov's 0-1 law, with probability 1 . By Theorem 1.1, this holds for every infinite cluster $C$ of $\omega$. 
Our main application of Theorem 1.1 is to prove in Section 4 a sufficient condition for having a unique infinite cluster. Define $\tau(x, y)$ to be the probability that $x$ and $y$ are in the same cluster. The process is said to exhibit connectivity decay if

$$
\inf \{\tau(x, y): x, y \in \mathrm{V}\}=0 .
$$

When a process does not have connectivity decay, it is said to exhibit long-range order. We show that for Bernoulli percolation (and some other percolation processes), nonuniqueness of the infinite cluster is equivalent to connectivity decay. One direction is easy; namely, if a.s. there is a unique infinite percolation cluster, then there is long-range order. This is an immediate consequence of Harris's inequality (that increasing events are positively correlated). Although the other direction seems intuitively obvious as well, its proof is surprisingly difficult (and it is still open for nonunimodular transitive graphs).

We note that connectivity decay in the nonuniqueness phase easily implies Theorem 3.2 of Schonmann (1999a) for the case of unimodular transitive graphs. (Our result also deals with percolation at $p_{u}$ and extends Schonmann's theorem to more general percolation processes, but his result extends to Bernoulli percolation on nonunimodular transitive graphs.)

REMARK 1.3. Fix a base vertex $o \in \mathrm{V}$ in $G$, and consider Bernoulli percolation in the nonuniqueness phase. Although $\inf \{\tau(o, v): v \in \mathrm{V}\}=0$, it may happen that

$$
\lim _{d \rightarrow \infty} \sup \{\tau(o, v): \operatorname{dist}(o, v)>d\}>0 .
$$

For example, take the Cayley graph of the free product $\mathbb{Z}^{2} * \mathbb{Z}_{2}$ with the usual generating set, that is, the Cayley graph corresponding to the presentation $\left\langle a, b, c \mid a b=b a, c=c^{-1}\right\rangle$. We have $p_{u}=1$, because the removal of any edge of the Cayley graph corresponding to the generator $c$ disconnects the graph. On the other hand, for $p>p_{c}\left(\mathbb{Z}^{2}\right)$, we have that all pairs of vertices in the same $\mathbb{Z}^{2}$ fiber (i.e., pairs $g, h$ such that $g^{-1} h$ is in the group generated by $a$ and $b$ ) are in the same cluster with probability bounded away from 0 .

Benjamini and Schramm (1996) asked for which Cayley graphs $G$ one has $p_{u}(G)<1$. It is easy to show that $p_{u}(G)=1$ when $G$ has more than one end. Babson and Benjamini (1999) showed that $p_{u}(G)<1$ when $G$ is a finitely presented group with one end. Häggström, Peres and Schonmann (1999) have shown that $p_{u}(G)<1$ if $G$ is a Cartesian product of infinite transitive graphs. Here, we show that this is also true of certain other classes of groups with one end, namely infinite Kazhdan groups (Corollary 6.6) and wreath products (Corollary 6.8). Presumably, all quasi-transitive (infinite) graphs with one end have $p_{u}<1$.

As another consequence of indistinguishability, we prove in Theorem 6.12 the inequalities $p_{u}\left(G \times H^{\prime}\right) \geq p_{u}(G \times H)$ and, in particular, $p_{u}(G) \geq p_{u}(G \times$ $H$ ), for Cayley graphs (or, more generally, unimodular transitive graphs) $G, H^{\prime}, H$ such that $G$ is infinite and $H^{\prime} \subset H$. 
Several other uses of cluster indistinguishability appear in Benjamini, Lyons, and Schramm (1999).

Crucial techniques for our proofs are the mass-transport principle and stationarity of delayed simple random walk, both explained below. These techniques were introduced in the study of percolation by Häggström (1997). In Section 5, we prove an ergodicity property for delayed random walk.

2. Background. We begin with some graph-theoretic terminology. Let $G=(\mathrm{V}(G), \mathrm{E}(G))$ be a graph with vertex set $\mathrm{V}(G)$ and (symmetric) edge set $\mathrm{E}(G) \subseteq \mathrm{V}(G) \times \mathrm{V}(G)$. When there is an edge in $G$ joining vertices $u, v$, we say that $u$ and $v$ are adjacent and write $u \sim v$. We always assume that the number of vertices adjacent to any given vertex is finite. The degree deg $v=$ $\operatorname{deg}_{G} v$ of a vertex $v \in \mathrm{V}(G)$ is the number of edges incident with it. A tree is a connected graph with no cycles. A forest is a graph whose connected components are trees. The distance between two vertices $u, v \in \mathrm{V}(G)$ is denoted by $\operatorname{dist}(v, u)=\operatorname{dist}_{G}(v, u)$ and is the least number of edges of a path in $G$ connecting $v$ and $u$. Given a set of vertices $K \subset \mathrm{V}(G)$, we let $\partial K$ denote its edge boundary, that is, the set of edges in $\mathrm{E}(G)$ having one vertex in $K$ and one outside of $K$. A transitive graph $G$ is amenable if inf $|\partial K| /|K|=0$, where $K$ runs over all finite nonempty vertex sets $K \subset \mathrm{V}(G)$.

An infinite set of vertices $V_{0} \subset \mathrm{V}(G)$ is end convergent if for every finite $K \subset \mathrm{V}(G)$, there is a component of $G-K$ that contains all but finitely many vertices of $V_{0}$. Two end-convergent sets $V_{0}, V_{1}$ are equivalent if $V_{0} \cup V_{1}$ is end convergent. An end of $G$ is an equivalence class of end-convergent sets. Let $\xi$ be an end of $G$. A neighborhood of $\xi$ is a set of vertices in $G$ that intersects every end-convergent set in $\xi$. In particular, when $K \subset \mathrm{V}(G)$ is finite, there is a component of $G-K$ that is a neighborhood of $\xi$.

Let $\Gamma$ be a finitely generated group and $S$ a finite symmetric generating set for $\Gamma$. Then the (right) Cayley graph $G=G(\Gamma, S)$ is the graph with vertices $\mathrm{V}(G):=\Gamma$ and edges $\mathrm{E}(G):=\{[v, v s]: v \in \Gamma, s \in S\}$.

Now suppose that $G=(\mathrm{V}(G), \mathrm{E}(G))$ is any graph, not necessarily a Cayley graph. An automorphism of $G$ is a bijection of $\mathrm{V}(G)$ with itself that preserves adjacency; $\operatorname{Aut}(G)$ denotes the group of all automorphisms of $G$ with the topology of pointwise convergence. If $\Gamma \subset \operatorname{Aut}(G)$, we say that $\Gamma$ is (vertex) transitive if for every $u, v \in \mathrm{V}(G)$, there is a $\gamma \in \Gamma$ with $\gamma u=v$. The graph $G$ is transitive if $\operatorname{Aut}(G)$ is transitive. The graph $G$ is quasi-transitive if $\mathrm{V}(G) / \operatorname{Aut}(G)$ is finite; that is, there is a finite set of vertices $V_{0}$ such that $\mathrm{V}(G)=\left\{\gamma v: \gamma \in \operatorname{Aut}(G), v \in V_{0}\right\}$. Note that any finitely generated group acts transitively on any of its Cayley graphs by the automorphisms $\gamma: v \mapsto \gamma v$.

It may seem that the most natural class of graphs on which percolation should be studied is the class of transitive (or quasi-transitive) locally finite graphs. At first sight, one might suspect that any theorem about percolation on Cayley graphs should hold for transitive graphs. However, somewhat surprisingly, this impression is not correct. It turns out that theorems about percolation on Cayley graphs "always" generalize to unimodular transitive 
graphs (to be defined shortly), but nonunimodular transitive graphs are quite different.

Recall that on every closed subgroup $\Gamma \subset \operatorname{Aut}(G)$, there is a unique (up to a constant scaling factor) Borel measure that, for every $\gamma \in \Gamma$, is invariant under left multiplication by $\gamma$; this measure is called (left) Haar measure. The group $\Gamma$ is unimodular if Haar measure is also invariant under right multiplication. For example, when $\Gamma$ is finitely generated and $G$ is the (right) Cayley graph of $\Gamma$, on which $\Gamma$ acts by left multiplication, then $\Gamma \subset \operatorname{Aut}(G)$ is (obviously) closed, unimodular and transitive. The Haar measure in this case is (a constant times) counting measure. A [quasi-]transitive graph $G$ is said to be unimodular if $\operatorname{Aut}(G)$ is unimodular. By Benjamini, Lyons, Peres and Schramm (1999) [hereinafter referred to as BLPS (1999)], a transitive graph is unimodular iff there is some unimodular transitive closed subgroup of $\operatorname{Aut}(G)$. It is not hard to show that a transitive closed subgroup $\Gamma \subset \operatorname{Aut}(G)$ is unimodular iff for all $x, y \in \mathrm{V}(G)$, we have

$$
\begin{aligned}
& \mid\{z \in \mathrm{V}(G): \exists \gamma \in \Gamma \gamma x=x \text { and } \gamma y=z\} \mid \\
& \quad=\mid\{z \in \mathrm{V}(G): \exists \gamma \in \Gamma \gamma y=y \text { and } \gamma x=z\} \mid
\end{aligned}
$$

[see Trofimov (1985)].

Here, unimodularity will be used only in the following form.

THEOREM 2.1 (Unimodular mass-transport principle). Let $G$ be a graph with a transitive unimodular closed automorphism group $\Gamma \subset \operatorname{Aut}(G)$. Let $o \in \mathrm{V}(G)$ be an arbitrary base point. Suppose that $\phi: \mathrm{V}(G) \times \mathrm{V}(G) \rightarrow[0, \infty]$ is invariant under the diagonal action of $\Gamma$. Then

$$
\sum_{v \in \mathrm{V}(G)} \phi(o, v)=\sum_{v \in \mathrm{V}(G)} \phi(v, o) .
$$

See BLPS (1999) for a discussion of this principle and for a proof. In fact, $\Gamma$ is unimodular iff (2.1) holds for every such $\phi$. Also see BLPS (1999) for further discussion of the relevance of unimodularity to percolation.

Given a set $A$, let $2^{A}$ be the collection of all subsets $\eta \subset A$, equipped with the $\sigma$-field generated by the events $\{a \in \eta\}$, where $a \in A$. A bond percolation process is a pair $(\mathbf{P}, \omega)$, where $\omega$ is a random element in $2^{\mathrm{E}}$ and $\mathbf{P}$ denotes the distribution (law) of $\omega$. Sometimes, for brevity, we shall just say that $\omega$ is a (bond) percolation. A site percolation process $(\mathbf{P}, \omega)$ is given by a probability measure $\mathbf{P}$ on $2^{\mathrm{V}(G)}$, while a (mixed) percolation is given by a probability measure on $2^{\mathrm{V}(G) \cup \mathrm{E}(G)}$ that is supported on subgraphs of $G$. If $\omega$ is a bond percolation process, then $\hat{\omega}:=\mathrm{V}(G) \cup \omega$ is the associated mixed percolation. In this case, we shall often not distinguish between $\omega$ and $\hat{\omega}$, and think of $\omega$ as a subgraph of $G$. Similarly, if $\omega$ is a site percolation, there is an associated mixed percolation $\hat{\omega}:=\omega \cup(\mathrm{E}(G) \cap(\omega \times \omega))$, and we shall often not bother to distinguish between $\omega$ and $\hat{\omega}$. 
If $v \in \mathrm{V}(G)$ and $\omega$ is a percolation on $G$, the cluster (or component) $C(v)$ of $v$ in $\omega$ is the set of vertices in $\mathrm{V}(G)$ that can be connected to $v$ by paths contained in $\omega$. We shall often not distinguish between the cluster $C(v)$ and the graph $(C(v),(C(v) \times C(v)) \cap \omega)$ whose vertices are $C(v)$ and whose edges are the edges in $\omega$ with endpoints in $C(v)$.

Let $p \in[0,1]$. Then Bernoulli $(p)$ bond percolation $\left(\mathbf{P}_{p}, \omega\right)$ on $G$ is the product measure on $2^{\mathrm{E}(G)}$ that satisfies $\mathbf{P}_{p}[e \in \omega]=p$ for all $e \in \mathrm{E}(G)$. Similarly, one defines Bernoulli $(p)$ site percolation on $2^{\mathrm{V}(G)}$. The critical probability $p_{c}(G)$ is the infimum over all $p \in[0,1]$ such that there is $\mathbf{P}_{p}$-positive probability for the existence of an infinite connected component in $\omega$.

Aizenman, Kesten and Newman (1987) showed that Bernoulli $(p)$ percolation in $\mathbb{Z}^{d}$ has a.s. at most one infinite cluster. Burton and Keane (1989) gave a much simpler argument that generalizes from $\mathbb{Z}^{d}$ to any amenable Cayley graph, though this generalization was not mentioned explicitly until Gandolfi, Keane and Newman (1992). It follows that $p_{u}(G)=p_{c}(G)$ when $G$ is an amenable Cayley graph. For background on percolation, especially in $\mathbb{Z}^{d}$, see Grimmett (1989).

Suppose that $\Gamma$ is an automorphism group of a graph $G$. A percolation process $(\mathbf{P}, \omega)$ on $G$ is $\Gamma$-invariant if $\mathbf{P}$ is invariant under each $\gamma \in \Gamma$. This is, of course, the case for Bernoulli percolation.

\section{Cluster indistinguishability.}

DEFINITION 3.1. Let $G$ be graph and $\Gamma$ a closed (vertex-) transitive subgroup of $\operatorname{Aut}(G)$. Let $(\mathbf{P}, \omega)$ be a $\Gamma$-invariant bond percolation process on $G$. We say that $\mathbf{P}$ has indistinguishable infinite clusters if for every measurable $\mathscr{A} \subset 2^{\mathrm{V}(G)} \times 2^{\mathrm{E}(G)}$ that is invariant under the diagonal action of $\Gamma$, almost surely, for all infinite clusters $C$ of $\omega$, we have $(C, \omega) \in \mathscr{A}$, or for all infinite clusters $C$, we have $(C, \omega) \notin \mathscr{A}$.

Definition 3.2. Let $G=(\mathrm{V}(G), \mathrm{E}(G))$ be a graph. Given a set $A \in$ $2^{\mathrm{E}(G)}$ and an edge $e \in \mathrm{E}(G)$, denote $\Pi_{e} A:=A \cup\{e\}$. For $\mathscr{A} \subset 2^{\mathrm{E}(G)}$, we write $\Pi_{e} \mathscr{A}:=\left\{\Pi_{e} A: A \in \mathscr{A}\right\}$. A bond percolation process $(\mathbf{P}, \omega)$ on $G$ is insertion tolerant if $\mathbf{P}\left[\Pi_{e^{e}} \mathscr{A}\right]>0$ for every $e \in \mathrm{E}(G)$ and every measurable $\mathscr{A} \subset 2^{\mathrm{E}(G)}$ satisfying $\mathbf{P}[\mathscr{A}]>0$.

For example, Bernoulli( $p$ ) bond percolation is insertion tolerant when $p \in(0,1]$.

A percolation $\omega$ is deletion tolerant if $\mathbf{P}\left[\Pi_{\left.\neg e^{\mathscr{A}}\right]}\right]>0$ whenever $e \in \mathrm{E}(G)$ and $\mathbf{P}[\mathscr{A}]>0$, where $\Pi_{\neg e} \omega:=\omega-\{e\}$. It turns out that deletion tolerance and insertion tolerance have very different implications. For indistinguishability of infinite clusters, we shall need insertion tolerance; deletion tolerance does not imply indistinguishability of infinite clusters (see Example 3.15 below). A percolation that is both insertion and deletion tolerant is usually said to have "finite energy." Gandolfi, Keane and Newman (1992) use the words "positive finite energy" in place of "insertion tolerance." 
THEOREM 3.3 (Cluster indistinguishability). Let $G$ be a graph with a transitive unimodular closed automorphism group $\Gamma \subset \operatorname{Aut}(G)$. Every $\Gamma$-invariant, insertion-tolerant, bond percolation process on $G$ has indistinguishable infinite clusters.

Similar statements hold for site and mixed percolations and the proofs go along the same lines. Likewise, the proof extends to quasi-transitive unimodular automorphism groups.

Initially, we could only establish the theorem under the assumption of strong insertion-tolerance; that is, $\mathbf{P}\left[\Pi_{e} \mathscr{A}\right] \geq \delta \mathbf{P}[\mathscr{A}]$ for some constant $\delta>0$. We are grateful to Olle Häggström for pointing out how to deal with the general case.

REMARK 3.4 (Scenery). For some purposes, the following more general form of this theorem is useful. Let $G$ be a graph and $\Gamma$ a transitive group acting on $G$. Suppose that $X$ is either $\mathrm{V}, \mathrm{E}$, or $\mathrm{V} \cup \mathrm{E}$. Let $Q$ be a measurable space and $\Omega:=2^{\mathrm{E}} \times Q^{X}$. A probability measure $\mathbf{P}$ on $\Omega$ will be called a bond percolation with scenery on $G$. The projection onto $2^{\mathrm{E}}$ is the underlying percolation and the projection onto $Q^{X}$ is the scenery. If $(\omega, q) \in \Omega$, we set $\Pi_{e}(\omega, q):=\left(\Pi_{e} \omega, q\right)$. We say that the percolation with scenery $\mathbf{P}$ is insertion-tolerant if, as before, $\mathbf{P}\left[\Pi_{e} \mathscr{B}\right]>0$ for every measurable $\mathscr{B} \subset \Omega$ of positive measure. We say that $\mathbf{P}$ has indistinguishable infinite clusters if for every $\mathscr{A} \subset 2^{\mathrm{V}} \times 2^{\mathrm{E}} \times Q^{X}$ that is invariant under the diagonal action of $\Gamma$, for P-a.e. $(\omega, q)$, either all infinite clusters $C$ of $\omega$ satisfy $(C, \omega, q) \in \mathscr{A}$ or they all satisfy $(C, \omega, q) \notin \mathscr{A}$. Theorem 3.3 holds also for percolation with scenery (with the same proof as given below).

A percolation with scenery that comes up naturally is as follows. Fix $p_{1}<p_{2}$ in $(0,1)$. Let $z_{e}(e \in \mathrm{E})$ be i.i.d. with each $z_{e}$ distributed according to uniform measure on $[0,1]$. Let $\omega_{j}$ be the set of $e \in \mathrm{E}$ with $z_{e}<p_{j}, j=1,2$. Then $\left(\omega_{2}, \omega_{1}\right)$ is an insertion-tolerant percolation with scenery, where $\omega_{1} \in 2^{\mathrm{E}}$ is considered the scenery. See Häggström and Peres (1999), Häggström, Peres and Schonmann (1999), Schonmann (1999a) and Alexander (1995) for examples where this process is studied.

Say that an infinite cluster $C$ is of type $\mathscr{A}$ if $(C, \omega) \in \mathscr{A}$; otherwise, say that it is of type $\neg \mathscr{A}$. Suppose that there is an infinite cluster $C$ of $\omega$ and an edge $e \in \mathrm{E}$ with $e \notin \omega$ such that the connected component $C^{\prime}$ of $\omega \cup\{e\}$ that contains $C$ has a type different from the type of $C$. Then $e$ is called pivotal for $(C, \omega)$.

We begin with an outline of the proof of Theorem 3.3. Assume that the theorem fails. First, we shall show that with positive probability, given that a vertex belongs to an infinite cluster, there are pivotal edges at some distance $r$ of the vertex. By Proposition 3.11, a.s. when there is more than one infinite cluster of $\omega$, each such cluster is transient for the simple random walk, and hence for the so-called delayed simple random walk (DSRW). The DSRW on a cluster of $\omega$ is stationary (in the sense of Lemma 3.13). Fix a base point $o \in \mathrm{V}$, and let $W$ be the DSRW on the $\omega$-cluster of $o$ with $W(0)=o$. Let $n$ be 
large, and let $e$ be a uniform random edge at distance $r$ from $W(n)$. When DSRW is transient, with probability bounded away from zero, $e$ is pivotal for $(C(o), \omega)$ and $W(j)$ is not an endpoint of $e$ for any time $j<n$. On this event, set $\omega^{\prime}:=\Pi_{e} \omega$. Then $\omega^{\prime}$ is, up to a controllable factor, as likely as $\omega$ by insertion tolerance. By transience, $e$ is far from $o$ with high probability. Since $e$ is pivotal, the type of $C(o)$ is different in $\omega$ and in $\omega^{\prime}$. Since $\omega$ and $\omega^{\prime}$ are the same in a large neighborhood of $o$, this shows that the type of $C(o)$ cannot be determined with arbitrary accuracy by looking at $\omega$ in a large neighborhood of $o$. This contradicts the measurability of $\mathscr{A}$ and establishes the theorem.

One can say that the proof is based on the contradictory prevalence of pivotal edges. To put the situation in the correct perspective, we point out that there are events depending on i.i.d. zero-one variables that have infinitely many "pivotals" with positive probability. For example, let $m_{1}, m_{2}, \ldots$ be a sequence of positive integers such that $\sum_{k} 2^{-m_{k}}<\infty$ but $\sum_{k} m_{k} 2^{-m_{k}}=\infty$, and let $\left\langle x_{k, j}: j \in\left\{1, \ldots, m_{k}\right\}\right\rangle$ be i.i.d. random variables with $\mathbf{P}\left[x_{k, j}=0\right]=$ $\mathbf{P}\left[x_{k, j}=1\right]=1 / 2$. Let $\mathscr{X}$ be the event that there is a $k$ such that $x_{k, j}=1$ for $j=1,2, \ldots, m_{k}$. Then with positive probability $\mathscr{X}$ has infinitely many pivotals; that is, there are infinitely many $(k, j)$ such that changing $x_{k, j}$ from 0 to 1 will change from $\neg \mathscr{X}$ to $\mathscr{X}$. [This is a minor variation on an example described by Häggström and Peres (private communication).]

We now prove the lemmas necessary for Theorem 3.3.

LEMMA 3.5 (Pivotals). Suppose that $(\mathbf{P}, \omega)$ is an insertion-tolerant percolation process on a graph $G$, and let $\mathscr{A} \subset 2^{\mathrm{V}(G)} \times 2^{\mathrm{E}(G)}$ be measurable. Assume that there is positive probability for coexistence of infinite clusters in $\mathscr{A}$ and $\neg \mathscr{A}$. Then with positive probability, there is an infinite cluster $C$ of the percolation that has a pivotal edge.

Proof. Let $k$ be the least integer such that there is positive probability that there are infinite clusters of different types with distance between them equal to $k$. Clearly, $k>0$. Suppose that $\gamma$ is a path of length $k$ such that with positive probability, $\gamma$ connects infinite clusters of different types; let $\mathscr{G}$ be the event that $\gamma$ connects infinite clusters of different types. Given $\mathscr{G}$, there are exactly two infinite clusters that intersect $\gamma$, by the minimality of $k$. Let $e$ be the first edge in $\gamma$. When $\omega \in \mathscr{G}$, let $C_{1}$ and $C_{2}$ be the two infinite clusters that $\gamma$ connects and let $C_{1}^{\prime}$ and $C_{2}^{\prime}$ be the infinite clusters of $\Pi_{e} \omega$ that contain $C_{1}$ and $C_{2}$, respectively. Note that, conditioned on $\mathscr{G}$, the distance between $C_{1}^{\prime}$ and $C_{2}^{\prime}$ is less than $k$. (The possibility that $C_{1}^{\prime}=C_{2}^{\prime}$ is allowed.) Since $\Pi_{e} \mathscr{G}$ has positive probability, the definition of $k$ ensures that the type of $\left(C_{1}^{\prime}, \Pi_{e} \omega\right)$ equals the type of $\left(C_{2}^{\prime}, \Pi_{e} \omega\right)$ a.s. Hence, $e$ is pivotal for $\left(C_{1}, \omega\right)$ or for $\left(C_{2}, \omega\right)$ whenever $\omega \in \mathscr{G}$. This proves the lemma.

LEMMA 3.6. Let $\Gamma$ be a transitive closed subgroup of the automorphism group of a graph $G$. If $(\mathbf{P}, \omega)$ is a $\Gamma$-invariant insertion-tolerant percolation process on an infinite graph $G$, then almost every ergodic component of $\omega$ is insertion tolerant. 
REMARK 3.7. A stronger and more general statement is Lemma 1 of Gandolfi, Keane and Newman (1992).

Proof of Lemma 3.6. This is the same as saying that for every $\Gamma$-invariant event $\mathscr{G}$ of positive probability, the probability measure $\mathbf{P}[\cdot \mid \mathscr{G}]$ is insertion tolerant. If $\mathscr{G}$ is a tail event, then $\Pi_{e} \mathscr{G}=\mathscr{G}$, and insertion tolerance of $\mathbf{P}[\cdot \mid \mathscr{G}]$ follows from the calculation

$$
\mathbf{P}\left[\Pi_{e} \mathscr{A} \mid \mathscr{G}\right]=\frac{\mathbf{P}\left[\Pi_{e} \mathscr{A} \cap \mathscr{G}\right]}{\mathbf{P}[\mathscr{G}]}=\frac{\mathbf{P}\left[\Pi_{e} \mathscr{A} \cap \Pi_{e} \mathscr{G}\right]}{\mathbf{P}[\mathscr{G}]}=\frac{\mathbf{P}\left[\Pi_{e}(\mathscr{A} \cap \mathscr{G})\right]}{\mathbf{P}[\mathscr{G}]} .
$$

But every $\Gamma$-invariant event is a tail event $(\bmod 0)$ : Write $E$ as an increasing union of finite subsets $\mathrm{E}_{n}$. Let $\mathscr{G}_{n}$ be events that do not depend on edges in $\mathrm{E}_{n}$ and such that $\sum_{n} \mathbf{P}\left[\mathscr{G}_{n} \triangle \mathscr{G}\right]<\infty$; such events exist because $\mathbf{P}$ and $\mathscr{G}$ are $\Gamma$-invariant. (Here $\mathscr{H} \triangle \mathscr{K}:=(\mathscr{H} \backslash \mathscr{K}) \cup(\mathscr{K} \backslash \mathscr{H})$ is the exclusive or.) Then $\lim \sup _{n} \mathscr{G}_{n}$ is a tail event and equals $\mathscr{G}(\bmod 0)$.

COROLlaRY 3.8. Let $\Gamma$ be a transitive closed subgroup of the automorphism group of a graph $G$. Consider a $\Gamma$-invariant insertion-tolerant percolation process $(\mathbf{P}, \omega)$ on $G$. Then almost surely, the number of infinite clusters of $\omega$ is 0,1 or $\infty$.

The proof is standard for the ergodic components; compare Newman and Schulman (1981).

Benjamini and Schramm (1996) conjectured that for Bernoulli percolation on any quasi-transitive graph, if there are infinitely many infinite clusters, then a.s. every infinite cluster has continuum many ends. This was proved by Häggström and Peres (1999) in the unimodular case and then by Häggström, Peres and Schonmann (1999) in general. For the unimodular case, we give a simpler proof that extends to insertion-tolerant percolation processes. We begin with the following proposition.

Proposition 3.9. Let $G$ be a graph with a transitive unimodular closed automorphism group $\Gamma \subset \operatorname{Aut}(G)$. Consider some $\Gamma$-invariant percolation process $(\mathbf{P}, \omega)$ on $G$. Then a.s. each infinite cluster that has at least three ends has no isolated ends.

PRoOF. For each $n=1,2, \ldots$, let $\hat{A}_{n}$ be the union of all vertex sets $A$ that are contained in some percolation cluster $K(A)$, have diameter at most $n$ in the metric of the percolation cluster and such that $K(A)-A$ has at least three infinite components. Note that if $\xi$ is an isolated end of a percolation cluster $K$, then for each finite $n$, some neighborhood of $\xi$ in $K$ is disjoint from $\hat{A}_{n}$. Also observe that if $K$ is a cluster with at least three ends, then $K$ intersects $\hat{A}_{n}$ for some $n$.

Fix some $n \geq 1$. Consider the mass transport that sends one unit of mass from each vertex $v$ in a percolation cluster that intersects $\hat{A}_{n}$ and distributes it equally among the vertices in $\hat{A}_{n}$ that are closest to $v$ in the metric of the 
percolation cluster of $v$. In other words, let $K(v)$ be the set of vertices in $C(v) \cap \hat{A}_{n}$, that are closest to $v$ in the metric of $\omega$, and set $F(v, w ; \omega):=$ $|K(v)|^{-1}$ if $w \in K(v)$ and otherwise $F(v, w ; \omega):=0$. Then $F(v, w ; \omega)$, and hence the expected mass $f(v, w):=\mathbf{E} F(v, w ; \omega)$ transported from $v$ to $w$, is invariant under the diagonal $\Gamma$ action. If $\xi$ is an isolated end of an infinite cluster $K$ that intersects $\hat{A}_{n}$, then there is a finite set of vertices $B$ that gets the mass from all the vertices in a neighborhood of $\xi$. But the mass-transport principle tells us that the expected mass transported to a vertex is finite. Hence, a.s. clusters that intersect $\hat{A}_{n}$ do not have isolated ends. Since this holds for all $n$, we gather that a.s. infinite clusters with isolated ends do not intersect $\cup_{n} \hat{A}_{n}$, whence they have at most two ends.

Proposition 3.10. Let $G$ be a graph with a transitive unimodular closed automorphism group $\Gamma \subset \operatorname{Aut}(G)$. If $(\mathbf{P}, \omega)$ is a $\Gamma$-invariant insertion-tolerant percolation process on $G$ with infinitely many infinite clusters a.s., then a.s. every infinite cluster has continuum many ends and no isolated end.

Proof. As Benjamini and Schramm (1996) noted, it suffices to prove that there are no isolated ends of clusters. To prove this in turn, observe that if some cluster has an isolated end, then because of insertion tolerance, with positive probability, some cluster will have at least three ends with one of them being isolated. Hence Proposition 3.10 follows from Proposition 3.9.

Proposition 3.11 (Transience). Let $G$ be a graph with a transitive unimodular closed automorphism group $\Gamma \subset \operatorname{Aut}(G)$. Suppose that $(\mathbf{P}, \omega)$ is a $\Gamma$-invariant insertion-tolerant percolation process on $G$ that has almost surely infinitely many infinite clusters. Then a.s. each infinite cluster is transient.

Proof. By Proposition 3.10, every infinite cluster of $\omega$ has infinitely many ends. Consequently, there is a random forest $\mathfrak{\lessgtr} \subset \omega$ whose distribution is $\Gamma$-invariant such that a.s. each infinite cluster $C$ of $\omega$ contains a tree of $\widetilde{F}$ with more than two ends [Lemma 7.4 of BLPS (1999)]. From Remark 7.3 of BLPS (1999), we know that any such tree has $p_{c}<1$. By Lyons (1990), it follows that such a tree is transient. The Rayleigh monotonicity principle [e.g., Lyons and Peres (1998)] then implies that $C$ is transient.

REMARK 3.12. Examples show that Proposition 3.11 does not hold when $\Gamma$ is not unimodular. However, we believe that when $\Gamma$ is not unimodular and $\mathbf{P}$ is Bernoulli percolation, it does still hold.

Let $(\mathbf{P}, \omega)$ be a bond percolation process on $G$, and let $\omega \in 2^{\mathrm{E}}$. Let $x \in \mathrm{V}(G)$ be some base point. It will be useful to consider delayed simple random walk on $\omega$ starting at $x, W=W_{x}^{\omega}$, defined as follows. Set $W(0):=x$. If $n \geq 0$, conditioned on $\langle W(0), \ldots, W(n)\rangle$ and $\omega$, let $W^{\prime}(n+1)$ be chosen from the neighbors of $W(n)$ in $G$ with equal probability. Set $W(n+1):=$ 
$W^{\prime}(n+1)$ if the edge $\left[W(n), W^{\prime}(n+1)\right]$ belongs to $\omega$; otherwise, let $W(n+1):=W(n)$.

Given $\omega$, let $W$ and $W^{*}$ be two independent delayed simple random walks starting at $x$. Set $w(n):=W(n)$ for $n \geq 0$ and $w(n):=W^{*}(-n)$ for $n<0$. Then $w$ is called two-sided delayed simple random walk. Let $\hat{\mathbf{P}}_{x}$ denote the law of the pair $(w, \omega)$; it is a probability measure on $\mathrm{V}^{\mathbb{Z}} \times 2^{\mathrm{E}}$. Define $\mathscr{S}$ : $\mathrm{V}^{\mathbb{Z}} \rightarrow \mathrm{V}^{\mathbb{Z}}$ by

$$
(\mathscr{S} w)(n):=w(n+1)
$$

and let

$$
\mathscr{S}(w, \omega):=(\mathscr{S} w, \omega) \quad \forall(w, \omega) \in \mathrm{V}^{\mathbb{Z}} \times 2^{\mathrm{E}} .
$$

For $\gamma \in \Gamma$, we set

$$
\gamma(w, \omega):=(\gamma w, \gamma \omega)
$$

where $(\gamma w)(n):=\gamma(w(n))$.

The following lemma generalizes similar lemmas in Häggström (1997), in Häggström and Peres (1999) and in Lyons and Peres (1998).

LEMMA 3.13 (Stationarity of delayed random walk). Let $G$ be a graph with a transitive unimodular closed automorphism group $\Gamma \subset \operatorname{Aut}(G)$. Let $o \in \mathrm{V}(G)$ be some base point. Let $(\mathbf{P}, \omega)$ be a $\Gamma$-invariant bond percolation process on $G$. Let $\hat{\mathbf{P}}_{o}$ be the joint law of $\omega$ and two-sided delayed simple random walk on $\omega$, as defined above. Then $\hat{\mathbf{P}}_{o}[\mathscr{A}]=\hat{\mathbf{P}}_{o}[\mathscr{S} \mathscr{A}]$ for every $\Gamma$-invariant $\mathscr{A} \subset \mathrm{V}^{\mathbb{Z}} \times 2^{\mathrm{E}}$. In other words, the restriction of $\hat{\mathbf{P}}_{o}$ to the $\Gamma$-invariant $\sigma$-field is $\mathscr{S}$-stationary.

The lemma will follow from two identities. The first is based on the fact that the transition operator for delayed simple random walk on $\omega$ is symmetric, and the second is based on the mass-transport principle, that is, on unimodularity.

Proof of Lemma 3.13. For $j \in \mathbb{Z}$ and $x \in \mathrm{V}$, set

$$
\mathscr{W}_{x}^{j}:=\left\{(w, \omega) \in \mathrm{V}^{\mathbb{Z}} \times 2^{\mathrm{E}}: w(j)=x\right\}
$$

and

$$
\mu:=\sum_{x \in \mathrm{V}} \hat{\mathbf{P}}_{x}
$$

Let $\mathscr{B} \subset \mathrm{V}^{\mathbb{Z}} \times 2^{\mathrm{E}}$ be measurable. Observe that for all $\omega \in 2^{\mathrm{E}}$,

$$
\mu[\mathscr{B} \mid \omega]=\mu[\mathscr{S} \mathscr{B} \mid \omega],
$$

where $\mu[\mathscr{B} \mid \omega]$ means $\sum_{x \in \mathrm{V}} \hat{\mathbf{P}}_{x}[\mathscr{B} \mid \omega]$. This follows from the fact that for any $j, k \in \mathbb{Z}$ with $j<0<k$ and any $v_{j}, v_{j+1}, \ldots, v_{k} \in \mathrm{V}$, we have

$$
\mu\left[\mathscr{W}_{v_{j}}^{j} \cap \mathscr{W}_{v_{j+1}}^{j+1} \cap \cdots \cap \mathscr{W}_{v_{k}}^{k} \mid \omega\right]=\prod_{i=j}^{k-1} a_{i},
$$

where $a_{i}:=\operatorname{deg}_{G}\left(v_{i}\right)^{-1}=\operatorname{deg}_{G}(o)^{-1}$ if $\left[v_{i}, v_{i+1}\right] \in \omega, a_{i}:=\left(\operatorname{deg}_{G}(o)-\right.$ $\left.\operatorname{deg}_{\omega}\left(v_{i}\right)\right) / \operatorname{deg}_{G}(o)$ if $v_{i}=v_{i+1}$ and $a_{i}:=0$ otherwise. By integrating (3.1) over 
$\omega$, we obtain

$$
\mu[\mathscr{B}]=\mu[\mathscr{S} \mathscr{B}],
$$

which is our first identity. Observe also that $\mu$ is $\Gamma$-invariant.

Now let $\mathscr{A} \subset \mathrm{V}^{\mathbb{Z}} \times 2^{\mathrm{E}}$ be $\Gamma$-invariant and measurable. For $x, y \in \mathrm{V}$, define

$$
\phi(x, y):=\mu\left[\mathscr{A} \cap \mathscr{W}_{x}^{-1} \cap \mathscr{W}_{y}^{0}\right] .
$$

Then $\phi$ is invariant under the diagonal action of $\Gamma$ on $\mathrm{V} \times \mathrm{V}$, because $\mu$ and $\mathscr{A}$ are $\Gamma$-invariant. Consequently, the mass-transport principle gives $\sum_{x \in \mathrm{V}} \phi(x, o)=\sum_{x \in \mathrm{V}} \phi(o, x)$, which translates to our second identity,

$$
\mu\left[\mathscr{A} \cap \mathscr{W}_{0}^{0}\right]=\mu\left[\mathscr{A} \cap \mathscr{W}_{o}^{-1}\right] .
$$

Observe that $\mu\left[\mathscr{C} \cap \mathscr{W}_{o}^{0}\right]=\hat{\mathbf{P}}_{o}[\mathscr{C}]$ for all measurable $\mathscr{C} \subset \mathrm{V}^{\mathbb{Z}} \times 2^{\mathrm{E}}$. By using (3.2) with $\mathscr{B}:=\mathscr{A} \cap \mathscr{W}_{0}^{0}$ and then using (3.3) with $\mathscr{S} \mathscr{A}$ in place of $\mathscr{A}$, we obtain finally

$$
\begin{aligned}
\hat{\mathbf{P}}_{o}[\mathscr{A}] & =\mu\left[\mathscr{A} \cap \mathscr{W}_{o}^{0}\right]=\mu\left[\mathscr{S}\left(\mathscr{A} \cap \mathscr{W}_{o}^{0}\right)\right] \\
& =\mu\left[\mathscr{S} \mathscr{A} \cap \mathscr{W}_{o}^{-1}\right]=\mu\left[\mathscr{S} \mathscr{A} \cap \mathscr{W}_{o}^{0}\right]=\hat{\mathbf{P}}_{o}[\mathscr{S} \mathscr{A}] .
\end{aligned}
$$

In Theorem 5.1 below, we show that in an appropriate sense, if $\omega$ is ergodic and has indistinguishable components, then the delayed simple random walk on the infinite components of $\omega$ is ergodic.

REMARK 3.14 (A generalization of Lemma 3.13). Let $\mathrm{V}$ be a countable set acted on by a transitive unimodular group $\Gamma$. Let $Q$ be a measurable space, let $\Xi:=Q^{\mathrm{V} \times \mathrm{V}}$, and let $\mathbf{P}$ be a $\Gamma$-invariant probability measure on $\Xi$. Suppose that $z: Q \rightarrow[0,1]$ is measurable, and that $z(\xi(x, y))=z(\xi(y, x))$ and $\sum_{v} z(\xi(x, v))=1$ for all $x, y \in \mathrm{V}$ and for P-a.e. $\xi$. Given $o \in \mathrm{V}$ and a.e. $\xi \in \Xi$, there is an associated random walk starting at $o$ with transition probabilities $p_{\xi}(x, y)=z(\xi(x, y))$. Let $\hat{\mathbf{P}}_{o}$ denote the joint distribution of $\xi$ and this random walk. Then the above proof shows that the restriction of $\hat{\mathbf{P}}_{o}$ to the $\Gamma$-invariant $\sigma$-field is $\mathscr{S}$-invariant.

See Lyons and Schramm (1999) for a still greater generalization.

Let $(\mathbf{P}, \omega)$ be a bond percolation process on an infinite graph $G$. For every $e \in \mathrm{E}(G)$, let $\mathscr{F}_{\neg e}$ be the $\sigma$-field generated by the events $\left\{e^{\prime} \in \omega\right\}$ with $e^{\prime} \neq e$. Set $Z(e):=\mathbf{P}\left[e \in \omega \mid \mathscr{F}_{\neg e}\right]$, and call this the conditional marginal of $e$. Note that insertion tolerance is equivalent to $Z(e)>0$ a.s. for every $e \in \mathrm{E}(G)$.

Proof of Theorem 3.3. Let $(\mathbf{P}, \omega)$ be insertion tolerant and $\Gamma$-invariant. Let $o \in \mathrm{V}$ be some fixed base point of $G$. Assume that the theorem is false. Then by Corollary 3.8, there is positive probability that $\omega$ has infinitely many infinite clusters. If we condition on this event, then $\omega$ is still insertion tolerant, as shown in Lemma 3.6. Consequently, we henceforth assume, with no loss of generality, that a.s. $\omega$ has infinitely many infinite clusters.

Fix $\varepsilon>0$. From Lemma 3.5, we know that there is a positive probability for pivotal edges of clusters of type $\mathscr{A}$, or there is a positive probability for 
pivotal edges of clusters of type $\neg \mathscr{A}$. Since we may replace $\mathscr{A}$ by its complement, assume, with no loss of generality, that there is a positive probability for pivotal edges of clusters of type $\mathscr{A}$. Fix some $r>0$ and $\delta>0$ such that with positive probability, $C(o)$ is infinite of type $\mathscr{A}$ and there is an edge $e$ at distance $r$ from $o$ that is pivotal for $(C(o), \omega)$ and satisfies $Z(e) \geq \delta$, where $Z(e)$ is the conditional marginal of $e$, as described above.

Let $\mathscr{A}_{o}$ be the event that $C(o)$ is infinite and $(C(o), \omega) \in \mathscr{A}$, and let $\mathscr{A}_{0}^{\prime}$ be an event that depends on only finitely many edges such that $\mathbf{P}\left[\mathscr{A}_{0} \triangle \mathscr{A}_{0}^{\prime}\right]<\varepsilon$. Let $R$ be large enough that $\mathscr{A}_{o}^{\prime}$ depends only on edges in the ball $B(o, R)$.

Let $W: \mathbb{Z} \rightarrow \bigvee$ be two-sided delayed simple random walk on $\omega$, with $W(0)=o$. For $n \in \mathbb{Z}$, let $e_{n} \in \mathrm{E}$ be an edge chosen uniformly among the edges at distance $r$ from $W(n)$. Write $\hat{\mathbf{P}}$ for the probability measure where we choose $\omega$ according to $\mathbf{P}$, choose $W$, and choose $\left\langle e_{n}: n \in \mathbb{Z}\right\rangle$ as indicated.

Given any $e \in \mathrm{E}$, let $\mathscr{P}_{e}$ be the event that $\omega \in \mathscr{A}_{o}$, that $e$ is pivotal for $C(o)$, and that $Z(e) \geq \delta$. Let $\mathscr{E}_{e}^{n}$ be the event that $e_{n}=e$ and $W(j)$ is not an endpoint of $e$ whenever $-\infty<j<n$. Note that for all $\zeta \in 2^{\mathrm{E}}, n \in \mathbb{Z}$, and $e \in \mathrm{E}$,

$$
\hat{\mathbf{P}}\left[\mathscr{E}_{e}^{n} \mid \omega=\zeta\right]=\hat{\mathbf{P}}\left[\mathscr{E}_{e}^{n} \mid \omega=\Pi_{e} \zeta\right] .
$$

Thus, for all measurable $\mathscr{B} \subset 2^{\mathrm{E}}$ with $\mathbf{P}[\mathscr{B}]>0$ and $\mathbf{P}[Z(e) \geq \delta \mid \mathscr{B}]=1$, we have

$$
\begin{aligned}
\hat{\mathbf{P}}\left[\mathscr{E}_{e}^{n} \cap \Pi_{e} \mathscr{B}\right] & =\hat{\mathbf{P}}\left[\mathscr{E}_{e}^{n} \mid \Pi_{e} \mathscr{B}\right] \mathbf{P}\left[\Pi_{e} \mathscr{B}\right]=\hat{\mathbf{P}}\left[\mathscr{E}_{e}^{n} \mid \mathscr{B}\right] \mathbf{P}\left[\Pi_{e} \mathscr{B}\right] \\
& =\frac{\mathbf{P}\left[\Pi_{e} \mathscr{B}\right]}{\mathbf{P}[\mathscr{B}]} \hat{\mathbf{P}}\left[\mathscr{E}_{e}^{n} \cap \mathscr{B}\right] \geq \delta \hat{\mathbf{P}}\left[\mathscr{E}_{e}^{n} \cap \mathscr{B}\right] .
\end{aligned}
$$

In particular,

$$
\hat{\mathbf{P}}\left[\mathscr{E}_{e}^{n} \cap \Pi_{e} \mathscr{A}_{0}^{\prime} \cap \Pi_{e} \mathscr{P}_{e}\right] \geq \delta \hat{\mathbf{P}}\left[\mathscr{E}_{e}^{n} \cap \mathscr{A}_{0}^{\prime} \cap \mathscr{P}_{e}\right]=\delta \hat{\mathbf{P}}\left[\mathscr{E}_{e}^{n} \cap \mathscr{A}_{0}^{\prime} \cap \mathscr{P}_{e_{n}}\right]
$$

Let

$$
\begin{aligned}
& \mathscr{E}^{n}:=\bigcup_{e \in \mathrm{E}} \mathscr{E}_{e}^{n}, \\
& \mathscr{E}_{R}^{n}:=\bigcup_{e \in \mathrm{E}-B(o, R)} \mathscr{E}_{e}^{n},
\end{aligned}
$$

and note that these are disjoint unions. Since $\Pi_{e} \mathscr{P}_{e} \subset \neg \mathscr{A}_{o}$ and since $\Pi_{e} \mathscr{A}_{o}^{\prime} \subset$ $\mathscr{A}_{o}^{\prime}$ when $e \notin B(o, R)$, we may sum (3.4) over all $e \notin B(o, R)$ to obtain that

$$
\begin{aligned}
\mathbf{P}\left[\mathscr{A}_{o}^{\prime} \cap \neg \mathscr{A}_{o}\right] & \geq \hat{\mathbf{P}}\left[\mathscr{E}_{R}^{n} \cap A_{o}^{\prime} \cap \neg \mathscr{A}_{o}\right] \geq \delta \hat{\mathbf{P}}\left[\mathscr{E}_{R}^{n} \cap \mathscr{A}_{o}^{\prime} \cap \mathscr{P}_{e_{n}}\right] \\
& \geq \delta \hat{\mathbf{P}}\left[\mathscr{E}_{R}^{n} \cap \mathscr{A}_{o} \cap \mathscr{P}_{e_{n}}\right]-\delta \varepsilon .
\end{aligned}
$$

Fix $n$ to be sufficiently large that the probability that $C(o)$ is infinite and $e_{n} \in B(o, R)$ is smaller than $\varepsilon$; this can be done by Proposition 3.11. Then $\hat{\mathbf{P}}\left[\mathscr{A}_{0} \cap \mathscr{E}^{n}-\mathscr{E}_{R}^{n}\right] \leq \varepsilon$. Hence, we have from (3.5) that

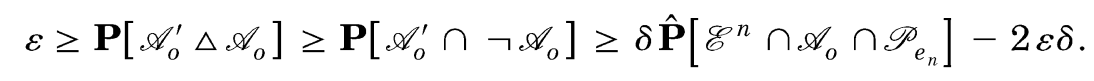


Recall that $\hat{\mathbf{P}}\left[\mathscr{A}_{0} \cap \mathscr{P}_{e_{0}}\right]>0$. Moreover, conditioned on $\mathscr{A}_{0} \cap \mathscr{P}_{e_{0}}$, transience guarantees a.s. a least $m \in \mathbb{Z}$ such that $W(m)$ is at distance $r$ to $e_{0}$. Consequently, for some $m \leq 0$,

$$
\hat{\mathbf{P}}\left[\mathscr{E}^{m} \cap \mathscr{A}_{0} \cap \mathscr{P}_{e_{m}}\right]>0 .
$$

Let $\mathscr{B}_{m}$ be the event that $Z\left(e_{m}\right) \geq \delta$, that $e_{m}$ is not an endpoint of $W(j)$ for $j<m$, that $C(W(m))$ is infinite and of type $\mathscr{A}$, and that $e_{m}$ is pivotal for $C(W(m))$. Then $\mathscr{B}_{m}=\mathscr{E}^{m} \cap \mathscr{A}_{0} \cap \mathscr{P}_{e_{m}}$. But $\mathscr{B}_{m}$ is $\Gamma$-invariant. Therefore, Lemma 3.13 shows that the left-hand side of (3.7) does not depend on $m$, and it certainly does not depend on $\varepsilon$. Hence, when we take $\varepsilon$ to be a sufficiently small positive number, (3.6) gives a contradiction. This completes the proof of the theorem.

EXAMPLE 3.15. A deletion-tolerant process that does not have indistinguishable clusters is obtained as follows. Let $X$ be a 3 -regular tree and $p \in(1 / 2,1)$. Let $p^{\prime} \in(1 /(2 p), 1)$. Begin with Bernoulli $(p)$ percolation on $X$. Independently for each cluster $C$, with probability $1 / 2$, intersect it with an independent Bernoulli $\left(p^{\prime}\right)$ percolation. The resulting percolation process is clearly deletion tolerant, yet some infinite clusters $\omega$ have $p_{c}(\omega)=1 /(2 p)$, while others have $p_{c}(\omega)=1 /\left(2 p p^{\prime}\right)$.

REMARK 3.16. Let $T$ be the 3-regular tree, let $\Gamma$ be the subgroup of automorphisms of $T$ that fixes an end $\xi$ of $T$ and let $\mathbf{P}$ be Bernoulli $(p)$ bond percolation on $T$, where $p \in(1 / 2,1)$. Then a.s. each infinite cluster $C$ has a unique vertex $v_{C}$ "closest" to $\xi$. The degree of $v_{C}$ in the percolation configuration distinguishes among the infinite clusters. Hence, Theorem 3.3 does not hold without the assumption that $\Gamma$ is unimodular. By a simple modification, a similar example can be constructed where $\Gamma$ is the full automorphism group of a graph on which the percolation is performed [compare BLPS (1999)].

However, Häggström, Peres and Schonmann (1999) have recently shown that even without the unimodularity assumption, when $p>p_{c}$ so-called "robust" properties do not distinguish between the infinite clusters of Bernoulli $(p)$ percolation.

QUESTION 3.17. In the case that $\Gamma$ is nonunimodular, write $\mu_{x}$ for the Haar measure of the stabilizer of $x \in \mathrm{V}$. The infinite clusters $C$ divide into two types: the heavy clusters for which $\sum_{x \in C} \mu_{x}=\infty$ and the others, the light clusters. It can be that the light clusters are distinguishable: for example, consider a 3-regular tree $T$ with a fixed end $\xi$. Let $\Gamma$ be the group of automorphisms of $T$ that fix $\xi$. Then Bernoulli(2/3) percolation has infinitely many light clusters, which can be distinguished by the degree of the vertex they contain that is closest to $\xi$. But is it the case that heavy clusters are indistinguishable for every insertion-tolerant percolation process that is invariant with respect to a transitive automorphism group? 
4. Uniqueness and connectivity. Our goal is to prove the following theorem.

THEOREM 4.1 (Uniqueness and connectivity). Let $G$ be an infinite graph with a transitive unimodular closed automorphism group $\Gamma \subset \operatorname{Aut}(G)$. Let $\mathbf{P}$ be a $\Gamma$-invariant and ergodic insertion-tolerant percolation process on $G$. If $\mathbf{P}$ has more than one infinite component a.s., then connectivity decays,

$$
\inf \{\tau(x, y): x, y \in \mathrm{V}\}=0 .
$$

The intuitive idea behind our proof is that if $\inf \tau(x, y)>0$, then each infinite cluster has a positive "density." Since the densities are the same by cluster indistinguishability, there are only finitely many infinite clusters. By Corollary 3.8, there is only one. To make the idea of "density" precise, we use simple random walk $X$ on the whole of $G$ with the percolation subgraph as the scenery, counting how many times we visit each cluster.

For a set $C \subset \mathrm{V}$, write

$$
\alpha(C):=\lim _{n \rightarrow \infty} \frac{1}{n} \sum_{k=1}^{n} \mathbf{1}_{\{X(k) \in C\}},
$$

where the limit exists, for the frequency of visits to $C$ by the simple random walk on $G$.

LEMмA 4.2 (Cluster frequencies). Let $G$ be a graph with a transitive unimodular closed automorphism group $\Gamma \subset \operatorname{Aut}(G)$. There is a $\Gamma$-invariant measurable function freq: $2^{\mathrm{V}} \rightarrow[0,1]$ with the following property. Suppose that $(\mathbf{P}, \omega)$ is a $\Gamma$-invariant bond percolation process on $G$, and let $\hat{\mathbf{P}}:=$ $\mathbf{P} \times \mathbf{P}_{o}$, where $\mathbf{P}_{o}$ is the law of simple random walk on $G$ starting at the base point o. Then $\hat{\mathbf{P}}$-a.s. $\alpha(C)=\operatorname{freq}(C)$ for every cluster $C$.

Proof. Given a set $C \subset \mathrm{V}$ and $m, n \in \mathbb{Z}, m<n$, let

$$
\alpha_{m}^{n}(C):=\frac{1}{n-m} \sum_{k=m}^{n-1} \mathbf{1}_{\{X(k) \in C\}} .
$$

For every $\alpha \in[0,1]$, let

$$
\mathscr{Z}_{\alpha}:=\left\{C \subset \mathrm{V}: \lim _{n \rightarrow \infty} \alpha_{0}^{n}(C)=\alpha \mathbf{P}_{o} \text {-a.s. }\right\},
$$

and set $\mathscr{Z}:=\bigcup_{\alpha \in[0,1]} \mathscr{Z}_{\alpha}$. Define freq $(C):=\alpha$ when $C \in \mathscr{Z}_{\alpha}$. If $C \notin \mathscr{Z}$, put freq $(C):=0$, say. It is easy to verify that freq is measurable. Let $\gamma \in \Gamma$. To prove that a.s. freq $(C)=\operatorname{freq}(\gamma C)$, note that there is an $m \in \mathbb{N}$ such that with positive probability $X(m)=\gamma o$. Hence for every measurable $A \subset[0,1]$ such that $\alpha(C) \in A$ with positive probability, we have $\alpha(\gamma C) \in A$ with positive probability. This implies that freq is $\Gamma$-invariant.

It remains to prove that $\mathbf{P}$-a.s., every component of $\omega$ is in $\mathscr{Z}$. First observe that the restriction of $\hat{\mathbf{P}}$ to the $\Gamma$-invariant $\sigma$-field is $\mathscr{S}$-invariant. This can be 
verified directly, but is also a special case of Remark 3.14: take $Q=\{-1,0,1\}$, take the value in $Q$ associated to a pair $(x, y) \in \mathrm{V} \times \mathrm{V}$ to be 1 if $[x, y] \in \omega, 0$ if $[x, y] \in \mathrm{E}-\omega$ and -1 if $[x, y] \notin \mathrm{E}$ and take $z(0)=z(1)=1 / \operatorname{deg}_{G}(o)$ and $z(-1)=0$.

The following argument is modeled on the proof of Theorem 1 of Burton and Keane (1989). Let $F^{n}(j)$ be the number of times that the $j$ most frequently visited clusters in $[0, n-1]$ are visited in $[0, n-1]$. That is,

$$
F^{n}(j):=n \max \left\{\alpha_{0}^{n}\left(C_{1}\right)+\cdots+\alpha_{0}^{n}\left(C_{j}\right): C_{1}, \ldots, C_{j} \text { are distinct clusters }\right\} .
$$

For each fixed $j$, it is easy to see that $F^{n}(j)$ is a subadditive sequence, that is, $F^{n+k}(j) \leq F^{n}(j)+\mathscr{S}^{n} F^{k}(j)$. Note that the random variables $F^{n}(j)$ are invariant with respect to the diagonal action of $\Gamma$ on $\mathrm{V}^{\mathbb{Z}} \times 2^{\mathrm{E}}$. By the subadditive ergodic theorem, $\lim _{n} F^{n}(j) / n$ exists a.s. Set $\alpha(j):=\lim _{n} F^{n}(j) / n-$ $\lim _{n} F^{n}(j-1) / n$ for $j \geq 1$. We claim that a.s.,

$$
\text { (4.1) } \lim _{n \rightarrow \infty} \max \left\{\left|\alpha_{0}^{m}(C)-\alpha_{0}^{k}(C)\right|: k, m \in \mathbb{Z}, k, m \geq n, C \text { is a cluster }\right\}=0
$$

the Cauchy property uniform in $C$. Indeed, let $\varepsilon>0$. Observe that $\sum_{j} \alpha(j) \leq 1$ and that $\alpha(1) \geq \alpha(2) \geq \cdots$. Let $j_{1} \geq 1$ be large enough that $\alpha(j)<\varepsilon / 9$ for all $j \geq j_{1}$. Let $m_{1}$ be sufficiently large that

$$
\left|F^{m}(j) / m-F^{m}(j-1) / m-\alpha(j)\right|<\varepsilon /\left(9 j_{1}+9\right)
$$

for all $j \leq j_{1}$ and all $m \geq m_{1}$. Set

$$
U:=\left\{x \in[0,1]: \exists j \leq j_{1}|x-\alpha(j)|<\varepsilon /\left(9 j_{1}+9\right)\right\} \cup[0, \varepsilon / 3],
$$

and let $U(\delta)$ denote the set of points $x \in \mathbb{R}$ within distance $\delta$ of $U$. For all clusters $C$ that are visited by the random walk and all $m=1,2, \ldots$, there is some $j$ such that $\alpha_{0}^{m}(C)=F^{m}(j) / m-F^{m}(j-1) / m$. If $j \leq j_{1}$ and $m \geq m_{1}$, it follows that $\alpha_{0}^{m}(C) \in U$. The same also holds when $j \geq j_{1}$ and $m \geq m_{1}$, because the $j_{1}$ th most frequently visited cluster in [0,m-1], say $C^{\prime}$, satisfies $\alpha_{0}^{m}(C) \leq \alpha_{0}^{m}\left(C^{\prime}\right) \leq \alpha\left(j_{1}\right)+\varepsilon / 9 \leq \varepsilon / 3$. Hence $\alpha_{0}^{m}(C) \in U$ for all clusters $C$ and all $m \geq m_{1}$. Because $-1 / m \leq \alpha_{0}^{m}(C)-\alpha_{0}^{m+1}(C) \leq 1 / m$, it follows that for all $C$ and all $n \geq m_{1}$, the set $\left\{\alpha_{0}^{m}(C): m \geq n\right\}$ is contained in some connected component of $U(1 / n)$. But when $n>\max \left\{m_{1}, 9\left(j_{1}+1\right) / \varepsilon\right\}$, the total length of $U(1 / n)$ is less than $\varepsilon$, which implies that the diameter of each connected component is less than $\varepsilon$. This verifies (4.1).

Since

$$
\begin{aligned}
& 2 \max \left\{\left|\alpha_{0}^{2 n}(C)-\alpha_{0}^{n}(C)\right|: C \text { is a cluster }\right\} \\
& \quad=\max \left\{\left|\alpha_{n}^{2 n}(C)-\alpha_{0}^{n}(C)\right|: C \text { is a cluster }\right\}
\end{aligned}
$$

has the same law as $\max \left\{\left|\alpha_{0}^{n}(C)-\alpha_{-n}^{0}(C)\right|: C\right.$ is a cluster $\}$ (by the $\mathscr{S}$-invariance noted above), it follows from (4.1) that a.s. $\lim _{n \rightarrow \infty} \alpha_{0}^{n}(C)=$ $\lim _{n \rightarrow \infty} \alpha_{-n}^{0}(C)$ for every cluster $C$. When the cluster $C$ is fixed, $\alpha_{0}^{n}(C)$ and $\alpha_{-n}^{0}(C)$ are independent, but both tend to $\alpha(C)$. Hence $\alpha(C)$ is an a.s. constant, which means that $\mathbf{P}$-a.s. we have $C \in \mathscr{Z}$ for every cluster $C$. This completes the proof.

Proof of Theorem 4.1. Let freq be as in Lemma 4.2. Since $\mathbf{P}$ is ergodic, Theorem 3.3 implies that there is a constant $c \in[0,1]$ such that a.s. freq $(C)$ 
$=c$ for every infinite cluster $C$. Suppose that there is more than one infinite cluster with positive probability. Then there are infinitely many infinite clusters a.s. Since clearly $\Sigma_{C} \alpha(C)=\Sigma_{C}$ freq $(C) \leq 1$, where the sum is over all clusters, it follows that $c=0$. Since $G$ is infinite, it is also immediate that freq $(\{v\})=0$ for every $v \in \mathrm{V}$. Therefore, freq $(C)=0$ a.s. for all clusters, finite or infinite. In particular, freq $(C(o))=0$ a.s., where $C(o)$ is the cluster of $o$.

Let $\tau_{0}:=\inf \{\tau(x, y): x, y \in \mathrm{V}\}$. We have that

$$
\mathbf{E}\left[\alpha_{0}^{n}(C(o))\right] \geq \tau_{0},
$$

whence $0=\mathbf{E}[\operatorname{freq}(C(o))]=\mathbf{E}[\alpha(C(o))] \geq \tau_{0}$ by the bounded convergence theorem, as required.

QUESTION 4.3. The proof actually shows that $(1 / n) \sum_{k=0}^{n-1} \mathbf{E}[\tau(o, X(k))] \rightarrow 0$ as $n \rightarrow \infty$ when there are infinitely many infinite clusters in an invariant percolation process that has indistinguishable clusters. Do we have $\tau(o, X(n)) \rightarrow 0$ a.s.?

EXAMPLE 4.4. We give an example of an ergodic invariant deletion-tolerant percolation process with infinitely many infinite clusters and with $\tau$ bounded below. The percolation process $\omega$ will take place on $\mathbb{Z}^{3}$. Let $\{a(n): n \in \mathbb{Z}\}$ be independent $\{0,1\}$-valued random variables with $\mathbf{P}[a(n)=1]=1 / 2$, and let $A$ be the set of vertices $\left(x_{1}, x_{2}, x_{3}\right) \in \mathbb{Z}^{3}$ with $a\left(x_{1}\right)=1$. Let $\varepsilon>0$ be small, and let $z_{e}$ be i.i.d. uniformly distributed in $[0,1]$ indexed by the edges of $\mathbb{Z}^{3}$ and independent of the $a(n)$ 's. Let $\omega_{1}$ be the set of edges $e$ with both endpoints in $A$ such that $z_{e}<1-\varepsilon$, and let $\omega_{2}$ be the set of edges $e$ with $z_{e}<\varepsilon$. Let $\omega_{3}$ be the set of edges that have no vertex in common with $\omega_{2} \cup A$ and satisfy $z_{e}<1-\varepsilon$. Set $\omega:=\omega_{1} \cup \omega_{2} \cup \omega_{3}$.

It is immediate to verify that when $\varepsilon$ is sufficiently small, a.s. $\omega$ has a single infinite component whose intersection with each component of $A$ is infinite, and has a single infinite component in each component of the complement of $A$. The claimed properties of this example follow easily.

5. Ergodicity of delayed random walk. The following theorem is not needed for the rest of the paper. It is presented here because its proof uses some of the ideas from the proof of Lemma 4.2.

THEOREM 5.1 (Ergodicity of delayed random walk). Let $G$ be a graph with a transitive unimodular closed automorphism group $\Gamma \subset \operatorname{Aut}(G)$. Let $o \in \mathrm{V}(G)$ be some base point. Let $(\mathbf{P}, \omega)$ be a $\Gamma$-invariant ergodic bond percolation process on $G$ with infinite clusters a.s. Also suppose that $\omega$ has indistinguishable infinite clusters a.s. Let $\hat{\mathbf{P}}_{o}$ be the joint law of $\omega$ and two-sided delayed simple random walk on $\omega$, starting at $o$. Let $\mathscr{A}$ be an event that is $\Gamma$-invariant and $\mathscr{S}$-invariant. Then $\hat{\mathbf{P}}_{o}[\mathscr{A} \mid C(o)$ is infinite $]$ is either 0 or 1.

Proof. For each $m, n \in \mathbb{Z}$, let $\mathscr{F}_{m}^{n}$ be the $\sigma$-field of $\Gamma$-invariant sets generated by $\omega$ and the random variables $\langle W(j): j \in[m, n]\rangle$, where $W(j)$ is the location of the delayed random walk at time $j$. 
Let $\mathscr{C}$ be the event that $C(o)$ is infinite. Let $\varepsilon>0$. Then there is an $n \in \mathbb{N}$ and an event $\mathscr{A}^{\prime} \in \mathscr{F}_{-n}^{n}$ such that $\hat{\mathbf{P}}_{o}\left[\mathscr{A}^{\prime} \Delta \mathscr{A}\right]<\varepsilon \mathbf{P}[\mathscr{C}]$, and therefore

$$
\hat{\mathbf{P}}_{o}\left[\mathscr{A}^{\prime} \triangle \mathscr{A} \mid \mathscr{C}\right]<\varepsilon .
$$

Note that $\mathscr{S} \mathscr{C}=\mathscr{C}$. Hence we have for all $m \in \mathbb{Z}$,

$$
\hat{\mathbf{P}}_{o}\left[\mathscr{S}^{m} \mathscr{A}^{\prime} \Delta \mathscr{A} \mid \mathscr{C}\right]<\varepsilon .
$$

Observe also that the two events $\mathscr{S}^{n+1} \mathscr{\mathscr { A }}^{\prime}$ and $\mathscr{S}^{-n-1} \mathscr{A}^{\prime}$ are independent given $\omega$ by the Markov property for the delayed random walk. Consequently,

$$
\begin{aligned}
\int_{\omega \in \mathscr{C}} \hat{\mathbf{P}}_{o}[\mathscr{A} \mid \omega]^{2} d \mathbf{P}[\omega] & \geq \int_{\omega \in \mathscr{C}} \hat{\mathbf{P}}_{o}\left[\mathscr{S}^{n+1} \mathscr{A}^{\prime} \mid \omega\right] \hat{\mathbf{P}}_{o}\left[\mathscr{S}^{-n-1} \mathscr{A}^{\prime} \mid \omega\right] d \mathbf{P}[\omega]-2 \varepsilon \\
& =\int_{\omega \in \mathscr{C}} \hat{\mathbf{P}}_{o}\left[\mathscr{S}^{n+1} \mathscr{\mathscr { A }}^{\prime}, \mathscr{S}^{-n-1} \mathscr{\mathscr { A }}^{\prime} \mid \omega\right] d \mathbf{P}[\omega]-2 \varepsilon \\
& \geq \int_{\omega \in \mathscr{C}} \hat{\mathbf{P}}_{o}[\mathscr{A} \mid \omega] d \mathbf{P}[\omega]-4 \varepsilon
\end{aligned}
$$

Since $\varepsilon$ is arbitrary, it follows that

$$
\int_{\mathscr{C}} \hat{\mathbf{P}}_{o}[\mathscr{A} \mid \omega]^{2} d \mathbf{P}[\omega] \geq \int_{\mathscr{C}} \hat{\mathbf{P}}_{o}[\mathscr{A} \mid \omega] d \mathbf{P}[\omega],
$$

which means that $\hat{\mathbf{P}}_{o}[\mathscr{A} \mid \omega]$ is 0 or 1 for almost every $\omega \in \mathscr{C}$.

Let $\mathscr{B}$ be the set of $\omega \in \mathscr{C}$ such that $\hat{\mathbf{P}}_{o}[\mathscr{A} \mid \omega]=1$ and let

$$
\hat{\mathscr{B}}:=\{(C(o), \omega): \omega \in \mathscr{B}\} .
$$

Finally, let $\Gamma \hat{\mathscr{B}}$ denote the orbit of $\hat{\mathscr{B}}$ under $\Gamma$; that is, $\Gamma \hat{\mathscr{B}}:=\{(C(\gamma o), \gamma \omega)$ : $\omega \in \mathscr{B}\}$. Because $\mathscr{S} \mathscr{A}=\mathscr{A}$ and $\mathscr{A}$ is $\Gamma$-invariant, it follows as in the beginning of the proof of Lemma 4.2 that for every $\gamma \in \Gamma$ and every $\omega$ such that $\gamma o \in C(o)$, we have $\omega \in \mathscr{B}$ iff $\gamma^{-1} \omega \in \mathscr{B}$ (after possibly making a measure zero modification of $\mathscr{B})$. Therefore,

$$
\Gamma \hat{\mathscr{B}} \cap\{(C, \omega): o \in C\}=\hat{\mathscr{B}} .
$$

Since $\omega$ is ergodic and has indistinguishable infinite clusters, a.s. all infinite clusters of $\omega$ are in $\Gamma \hat{\mathscr{B}}$ or a.s. all infinite clusters of $\omega$ are not in $\Gamma \hat{\mathscr{B}}$. If the latter is the case, then $\mathbf{P}[\mathscr{B}]=0$ and hence $\hat{\mathbf{P}}_{o}[\mathscr{A} \mid \mathscr{C}]=0$. Therefore, assume that

$$
\mathbf{P}[(C(o), \omega) \in \Gamma \hat{\mathscr{B}} \mid \mathscr{C}]=1 .
$$

Hence, by (5.2), $\mathbf{P}[\mathscr{B} \mid \mathscr{C}]=1$, giving $\hat{\mathbf{P}}_{o}[\mathscr{A} \mid \mathscr{C}]=1$, as required.

6. Uniqueness for Bernoulli percolation. We now present some applications of Theorem 4.1 to Bernoulli percolation on Cayley graphs. Later in this section, we prove inequalities relating $p_{u}$ based on Theorem 3.3.

We first note that the choice of generators does not influence whether $p_{u}<1$. 
THEOREM 6.1. Let $S_{1}$ and $S_{2}$ be two finite generating sets for a countable group $\Gamma$, yielding corresponding Cayley graphs $G_{1}$ and $G_{2}$. Then $p_{u}\left(G_{1}\right)<1$ iff $p_{u}\left(G_{2}\right)<1$.

Proof. Left and right Cayley graphs with respect to a given set of generators are isomorphic via $x \mapsto x^{-1}$, so we consider only right Cayley graphs.

Write $\tau_{p}^{i}(x)$ for the probability under Bernoulli( $p$ ) percolation that $o$ and $x$ lie in the same cluster of $G_{i}$. Express each element $s \in S_{1}$ in terms of a word $\varphi(s) \in S_{2}$. Let $\omega_{2}$ be Bernoulli $(p)$ percolation on $G_{2}$ and define $\omega_{1}$ on $G_{1}$ by letting $[x, x s] \in \omega_{1}$ iff the path from $x$ to $x s$ in $G_{2}$ given by $\varphi(s)$ lies in $\omega_{2}$. Then $\omega_{1}$ is a percolation such that if two edges are sufficiently far apart, then their presence in $\omega_{1}$ is independent. Thus, Liggett, Schonmann and Stacey (1997) provide a function $f(p) \in(0,1)$ such that $f(p) \uparrow 1$ when $p \uparrow 1$ and such that $\omega_{1}$ stochastically dominates Bernoulli $(f(p))$ percolation on $G_{1}$. This implies that $\tau_{f(p)}^{1}(x) \leq \tau_{p}^{2}(x)$ for each $x$. If $f(p)>p_{u}\left(G_{1}\right)$, then it follows from this and Theorem 4.1 that $p \geq p_{u}\left(G_{2}\right)$, showing that $p_{u}\left(G_{1}\right)<1$ implies $p_{u}\left(G_{2}\right)<1$.

REMARK 6.2. For the situation used in the above proof, and for many similar applications, one does not need the full generality of the theorem of Liggett, Schonmann and Stacey. The following observation suffices. Suppose that $\left(X_{i}: i \in I\right)$ are i.i.d. random variables taking values in $\{0,1\}$ and with $\mathbf{P}\left[X_{i}=1\right]=p$. Let $\left(I_{j}: j \in J\right)$ be an indexed collection of subsets of $I$. Set $Y_{j}:=\min \left\{X_{i}: i \in I_{j}\right\}$ and $J_{i}:=\left\{j \in J: i \in I_{j}\right\}$. Suppose that $n:=\sup \left\{\left|I_{j}\right|: j \in\right.$ $J\}$ and $m:=\sup \left\{\left|J_{i}\right|: i \in I\right\}$ are both finite. Then $\left(Y_{j}: j \in J\right)$ stochastically dominates independent random variables $\left(Z_{j}: j \in J\right)$ with $\mathbf{P}\left[Z_{j}=1\right] \geq(1-$ $\left.(1-p)^{1 / m}\right)^{n}$. Indeed, set $X_{i}^{\prime}:=\max \left\{Z_{i, j}: j \in J_{i}\right\}$ and $Z_{j}:=\min \left\{Z_{i, j}: i \in I_{j}\right\}$, where $\left(Z_{i, j}: i \in I, j \in J\right)$ are independent $\{0,1\}$-variables with $\mathbf{P}\left[Z_{i, j}=1\right]=$ $1-(1-p)^{1 / m}$. Then $\left(X_{i}: i \in I\right)$ stochastically dominates $\left(X_{i}^{\prime}: i \in I\right)$, and hence $\left(Y_{j}: j \in J\right)$ stochastically dominates $\left(Z_{j}: j \in J\right)$.

REMARK 6.3. Schonmann (1999a) shows that for every quasi-transitive graph $G, p_{u}=p_{B B}$, where $p_{B B}$ is the infimum of all $p$ such that

$$
\lim _{r \rightarrow \infty} \inf _{v, u \in \mathrm{V}} \mathbf{P}[B(v, r) \leftrightarrow B(u, r)]=1
$$

in Bernoulli $(p)$ percolation, where $B(v, r)$ denotes the ball in $G$ of radius $r$ and center $v$ and $A \leftrightarrow A^{\prime}$ is the event that there is a cluster $C$ with $C \cap A \neq \varnothing$ and $C \cap A^{\prime} \neq \varnothing$. Based on this and the proof of Theorem 6.1, one obtains the following generalization. Suppose that $G$ and $G^{\prime}$ are quasi-transitive graphs and $G^{\prime}$ is quasi-isometric to $G$. Then $p_{u}(G)<1$ iff $p_{u}\left(G^{\prime}\right)<1$. This observation was also made independently by Y. Peres (private communication).

For our next result regarding $p_{u}$, we need the following construction. Let $\kappa_{0}$ be a real-valued random variable and suppose that $\mathbf{P}$ is a bond percolation 
process on some graph $G$. We would like to color the clusters of $\mathbf{P}$ in such a way that conditioned on the configuration $\omega$, the colors of the components are i.i.d. random variables with the same law as $\kappa_{0}$.

To construct this process, let $\left\langle v_{1}, v_{2}, \ldots\right\rangle$ be an ordering of the vertices in $G$. Let $\left\langle\kappa_{1}, \kappa_{2}, \ldots\right\rangle$ be i.i.d. random variables with the same law as $\kappa_{0}$. Given $\omega$ and $v \in \mathrm{V}$, set $\kappa_{\omega}(v):=\kappa_{j}$ if $j$ is the least integer with $v_{j} \in C(v)$. It is not hard to see that $\kappa_{\omega}(v)$ is measurable, as a function on the product of $2^{\mathrm{E}}$ and the sample spaces of the $\kappa_{j}$ 's. Let $\mathbf{P}^{\kappa}$ denote the law of $\left(\omega, \kappa_{\omega}\right)$. Observe that $\mathbf{P}^{\kappa}$ satisfies the description of the previous paragraph, and therefore does not depend on the choice of the ordering of $\mathrm{V}$. Consequently, if $\gamma$ is an automorphism of $G$ and $\mathbf{P}$ is $\gamma$-invariant, then $\mathbf{P}^{\kappa}$ is $\gamma$-invariant.

LEMMA 6.4 (Ergodicity of $\mathbf{P}^{\kappa}$ ). Let $\Gamma$ be a transitive closed subgroup of the automorphism group of a graph $G$. Suppose that $(\mathbf{P}, \omega)$ is a $\Gamma$-invariant ergodic insertion-tolerant percolation process on $G$. Let $\kappa_{0}$ be a real-valued random variable, and let $\mathbf{P}^{\kappa}$ be as above. Suppose that $\inf \{\tau(o, x): x \in \mathrm{V}(G)\}$ $=0$. Then $\mathbf{P}^{\kappa}$ is $\Gamma$-invariant and ergodic.

Proof. To prove the ergodicity of $\mathbf{P}^{\kappa}$, let $\mathscr{A}$ be a $\Gamma$-invariant event in $2^{\mathrm{E}(G)} \times \mathbb{R}^{\mathrm{V}(G)}$ and $\varepsilon \in(0,1 / 2)$. The probability of $\mathscr{A}$ conditioned on $\omega$ must be a constant, say $a$, by ergodicity of $\mathbf{P}$. We need to show that $a \in\{0,1\}$.

There is a cylindrical event $\mathscr{A}^{\prime}$ that depends only on the restriction of $\omega$ and $\kappa_{\omega}$ to some ball $B(o, r)$ about $o$ and such that $\mathbf{P}^{\kappa}\left[\mathscr{A} \Delta \mathscr{A}^{\prime}\right]<\varepsilon$. Then $\mathbf{E}\left[\mathbf{P}^{\kappa}\left[\mathscr{A}^{\prime} \mid \omega\right]-a \mid\right]<\varepsilon$.

Let $\mathscr{B}_{x}$ be the event that some vertex in $B(o, r)$ belongs to the same cluster as some vertex in $B(x, r)$. Since $\inf _{x} \tau(o, x)=0$ and $\mathbf{P}$ is insertion tolerant, there is some $x$ such that $\mathbf{P}\left[\mathscr{B}_{x}\right]<\varepsilon$. Indeed, suppose that $\mathbf{P}\left[\mathscr{B}_{x}\right] \geq$ $\varepsilon$ for all $x$. Let $\mathscr{D}_{x}$ be the event that all the edges in $B(x, r)$ belong to $\omega$, and for $A \subset G$, let $\mathscr{F}_{\neg A}$ denote the $\sigma$-field generated by the events $\{e \in \omega\}$ with $e \notin A$. Then $\mathscr{B}_{x}$ is $\mathscr{F}_{\neg(B(o, r) \cup B(x, r))}$-measurable. By insertion tolerance, for every $\mathscr{F}_{\neg B(o, r)}$-measurable event $\mathscr{C}$ with $\mathbf{P}[\mathscr{C}]>0$, we have $\mathbf{P}\left[\mathscr{C} \cap \mathscr{D}_{0}\right]>0$. Consequently, there is some $\delta>0$ such that

$$
\mathbf{P}\left[\mathbf{P}\left[\mathscr{D}_{o} \mid \mathscr{F}_{\neg B(o, r)}\right]>\delta\right]>1-\varepsilon / 2 .
$$

It follows that for all $\mathscr{F}_{\neg B(o, r)}$-measurable events $\mathscr{C}$ with $\mathbf{P}[\mathscr{C}] \geq \varepsilon$, we have $\mathbf{P}\left[\mathscr{D}_{o} \mid \mathscr{C}\right]>\delta / 2$. In particular, for all $x \in \mathrm{V}$, we have $\mathbf{P}\left[\mathscr{D}_{o} \mid \mathscr{B}_{x}\right]>\delta / 2$, which gives $\mathbf{P}\left[\mathscr{D}_{0} \cap \mathscr{B}_{x}\right]>\varepsilon \delta / 2$. Transitivity and insertion tolerance imply that there is a $\delta^{\prime}>0$ such that

$$
\mathbf{P}\left[\mathbf{P}\left[\mathscr{D}_{x} \mid \mathscr{F}_{\neg B(x, r)}\right]>\delta^{\prime}\right]>1-\varepsilon \delta / 4
$$

for all $x \in \mathrm{V}$. Hence, for all $\mathscr{F}_{\neg B(x, r)}$-measurable events $\mathscr{C}$ with $\mathbf{P}[\mathscr{C}] \geq \varepsilon \delta / 2$, we have $\mathbf{P}\left[\mathscr{D}_{x} \mid \mathscr{C}\right]>\delta^{\prime} / 2$. Taking $\mathscr{C}:=\mathscr{D}_{o} \cap \mathscr{B}_{x}$ gives for all $x \in \mathrm{V}-$ $B(o, 2 r)$,

$$
\tau(o, x) \geq \mathbf{P}\left[\mathscr{D}_{o} \cap \mathscr{B}_{x} \cap \mathscr{D}_{x}\right]=\mathbf{P}\left[\mathscr{D}_{x} \mid \mathscr{D}_{o} \cap \mathscr{B}_{x}\right] \mathbf{P}\left[\mathscr{D}_{o} \cap \mathscr{B}_{x}\right] \geq\left(\delta^{\prime} / 2\right)(\varepsilon \delta / 2),
$$


which contradicts our assumption, and thereby verifies that $\mathbf{P}\left[\mathscr{B}_{x}\right]<\varepsilon$ for some $x \in \mathrm{V}$. Fix such an $x$.

Let $\gamma_{x} \in \Gamma$ be such that $\gamma_{x} o=x$. Then

$$
\left.\mathbf{E}\left[\mathbf{P}^{\kappa}\left[\gamma_{x} \mathscr{A}^{\prime} \mid \omega\right]-a\right]\right]<\varepsilon .
$$

Since $\gamma_{x} \mathscr{A}^{\prime}$ depends only on the colored configuration in $B(x, r)$, we have that on the complement of $\mathscr{B}_{x}$,

$$
\mathbf{P}^{\kappa}\left[\mathscr{A}^{\prime}, \gamma_{x} \mathscr{A}^{\prime} \mid \omega\right]=\mathbf{P}^{\kappa}\left[\mathscr{A}^{\prime} \mid \omega\right] \mathbf{P}^{\kappa}\left[\gamma_{x} \mathscr{A}^{\prime} \mid \omega\right],
$$

whence $\mathbf{E}\left[\mathbf{P}^{\kappa}\left[\mathscr{A}^{\prime}, \gamma_{x} \mathscr{A}^{\prime} \mid \omega\right]-a^{2} \mid\right]=O(\varepsilon)$. Therefore,

$$
a-a^{2}=\mathbf{E}\left[\left|\mathbf{P}^{\kappa}\left[\mathscr{A}, \gamma_{x} \mathscr{A} \mid \omega\right]-a^{2}\right|\right]=O(\varepsilon) .
$$

Since $\varepsilon$ was arbitrary, we get that $a \in\{0,1\}$, as desired.

We now prove that $p_{u}<1$ for all Cayley graphs of Kazhdan groups. Let $\Gamma$ be a countable group and $S$ a finite subset of $\Gamma$. Let $\mathscr{U}(\mathscr{H C})$ denote the set of unitary representations of $\Gamma$ on a Hilbert space $\mathscr{H}$ that have no invariant vectors except 0 . Set

$$
\kappa(\Gamma, S):=\max \{\varepsilon: \forall \mathscr{H} \forall \pi \in \mathscr{U}(\mathscr{H}) \forall v \in \mathscr{H}, \exists s \in S\|\pi(s) v-v\| \geq \varepsilon\|v\|\} .
$$

Then $\Gamma$ is called Kazhdan [or has Kazhdan's property (T)] if $\kappa(T, S)>0$ for all finite $S$. The only amenable Kazhdan groups are the finite ones. Examples of Kazhdan groups include $\mathrm{SL}(n, \mathbb{Z})$ for $n \geq 3$. See de la Harpe and Valette (1989) for background; in particular, every Kazhdan group is finitely generated (page 11), but not necessarily finitely presentable (as shown by examples of Gromov; see page 43). It can be shown directly, but also follows from our Corollary 6.6 below, that every infinite Kazhdan group has only one end. See Zuk (1996) for examples of Kazhdan groups arising as fundamental groups of finite simplicial complexes.

Rather than the definition, we shall use the following characterization of Kazhdan groups: Let $\mathbf{P}_{*}$ be the probability measure on subsets of $\Gamma$ that is the empty set half the time and all of $\Gamma$ half the time. Recall that $\Gamma$ acts by translation on the probability measures on $2^{\Gamma}$.

THEOREM 6.5 [Glasner and Weiss (1997)]. A countable infinite group $\Gamma$ is Kazhdan iff $\mathbf{P}_{*}$ is not in the weak* closure of the $\Gamma$-invariant ergodic probability measures on $2^{\Gamma}$.

COROLLARY 6.6. If $G$ is a Cayley graph of an infinite Kazhdan group $\Gamma$, then $p_{u}(G)<1$. Moreover, $\mathbf{P}[\exists$ a unique infinite cluster $]=0$ in Bernoulli $\left(p_{u}\right)$ percolation.

In an older version of this manuscript, only the first statement appeared. We thank Yuval Peres for pointing out that a modification of the original proof produces the stronger second statement.

Schonmann (1999b) proved that Bernoulli $\left(p_{u}\right)$ on $T \times \mathbb{Z}$, where $T$ is a regular tree of degree at least three, does not have a unique infinite cluster, 
and Peres (1999) generalized this result to nonamenable products. On the other hand, Bernoulli $\left(p_{u}\right)$ percolation on a planar nonamenable transitive graph has a unique infinite cluster. [See Lalley (1998) for the high genus case, and Benjamini and Schramm (1998) for the general case.] Little else is known, however, about the uniqueness of infinite clusters at $p_{u}$. For example, the case of lattices in hyperbolic 3-space is still open.

Proof of Theorem 6.5. Suppose that $p_{u}(G)=1$. Let $o$ be the identity in $\Gamma$, regarded as a vertex in $G$. Write $\tau_{p}(x)$ for the probability under Bernoulli $(p)$ percolation that $o$ and $x$ lie in the same cluster. Then by Theorem 4.1, for all $p<1$ we have $\inf _{x} \tau_{p}(x)=0$. Fix $p$ and let $\omega$ be the open subgraph of a Bernoulli $(p)$ percolation. Let $\eta$ be the union of the sites of some of the clusters of $\omega$, where each cluster is independently put in $\eta$ with probability $1 / 2$. By Lemma 6.4, the law $\mathbf{Q}_{p}$ of $\eta$ is $\Gamma$-invariant and ergodic. Furthermore, any fixed finite subset of $\Gamma$ either is contained in $\eta$ or is disjoint from $\eta$ with high probability when $p$ is sufficiently close to 1 . That is, $\mathbf{P}_{*}=$ weak $^{*}$ $\lim _{p \rightarrow 1} \mathbf{Q}_{p}$, whence $\Gamma$ is not Kazhdan.

To prove the stronger statement, suppose that there is a unique infinite component $\mathbf{P}$-a.s. Let $(\omega, \tilde{\omega})$ be the standard coupling of $\operatorname{Bernoulli}(p)$ and Bernoulli $\left(p_{u}\right)$ percolation on $G$. Let $\eta$ be as above. For a vertex $x \in G$, write $A(x)$ for the set of clusters of $\omega$ that lie in the unique infinite cluster of $\tilde{\omega}$ and that are closest to $x$ among those with this property (where distance is measured in $G$ ). Define $\eta^{\prime}$ to be the union of $\eta$ with all sites $x$ for which $A(x)$ contains only one cluster and that cluster lies in $\eta$. Since the law of $(\omega, \tilde{\omega}, \eta)$ is ergodic by an obvious extension of Lemma 6.4, so is the law $\mathbf{Q}_{p}^{\prime}$ of its factor $\eta^{\prime}$. Again, we obtain $\mathbf{P}_{*}=$ weak*- $\lim _{p \rightarrow p_{u}} \mathbf{Q}_{p}^{\prime}$, whence $\Gamma$ is not Kazhdan.

REMARK 6.7. For probabilists, we believe that the proof we have presented of Corollary 6.6 is the most natural. For others, we note that one can avoid Lemma 6.4 and Theorem 6.5 by using a theorem of Delorme (1977) and Guichardet (1977) that characterizes Kazhdan groups in terms of positive semidefinite functions. This relies on the fact that $\tau(x, y)$ is positive semidefinite, as observed by Aizenman and Newman (1984).

Other groups that are not finitely presentable and that have provided interesting examples for probability theory are the so-called lamplighter groups [see Kaimanovich and Vershik (1983) and Lyons, Pemantle and Peres (1996); in these references, these groups are denoted $G_{d}$ and are amenable, but we shall be interested here in nonamenable examples].

We first give a concrete description of a lamplighter graph and later generalize and use more algebraic language. Suppose that $G$ is a graph. The lamplighter graph $L_{G}$ over $G$ is the graph whose vertices are pairs $(A, v)$, where $A \subset \mathrm{V}(G)$ is finite and $v \in \mathrm{V}(G)$. We think of $A$ as the locations of the lamps that are on, and consider $v$ as the location of the lamplighter. One neighbor of $(A, v)$ in $L_{G}$ is the vertex $(A \Delta\{v\}, v)$ (the lamplighter switches the lamp off or on) and the other neighbors have the form $(A, u)$, where $[v, u] \in \mathrm{E}(G)$ (the lamplighter walks one step). 
In the algebraic context and language, lamplighter groups are particular wreath products: Let $\Gamma$ be a group acting from the left on a set $\mathrm{V}$. Let $K$ be a group; $K_{*}^{\mathrm{V}}$ denotes the group of maps $f: \mathrm{V} \rightarrow K$ such that $f(x)$ is the identity element $\operatorname{id}_{K}$ of $K$ for all but finitely many $x \in \mathrm{V}$ and with multiplication $\left(f_{1} f_{2}\right)(x):=f_{1}(x) f_{2}(x)$. Then $\Gamma$ acts on $K_{*}^{\vee}$ by translation: $(\gamma f)(x):=f\left(\gamma^{-1} x\right)$. The (restricted) wreath product $K \backslash \Gamma$ is the set $K_{*}^{\vee} \times \Gamma$ with the multiplication

$$
\left(f_{1}, \gamma_{1}\right)\left(f_{2}, \gamma_{2}\right):=\left(f_{1}\left(\gamma_{1} f_{2}\right), \gamma_{1} \gamma_{2}\right) \text {. }
$$

If $\Gamma$ and $K$ are finitely generated and $\Gamma$ acts transitively on $V$, then $K \backslash \Gamma$ is finitely generated. To see this, let $\gamma_{1}, \ldots, \gamma_{s}$ generate $\Gamma$ and $k_{1}, \ldots, k_{t}$ generate $K$. Write $\mathrm{id}_{\Gamma}$ for the identity element of $\Gamma$ and $\operatorname{id}_{\vee}$ for the identity element of $K_{*}^{\vee}$. Let $o \in \mathrm{V}$ be fixed. Write $F_{j}$ for the element of $K_{*}^{\vee}$ defined by $F_{j}(o):=k_{j}$ and $F_{j}(x):=\mathrm{id}_{K}$ for all $x \neq o$. Set

$$
\begin{aligned}
& S_{\Gamma}:=\left\{\left(\operatorname{id}_{\mathrm{V}}, \gamma_{i}\right): 1 \leq i \leq s\right\}, \\
& S_{K}:=\left\{\left(F_{j}, \operatorname{id}_{\Gamma}\right): 1 \leq j \leq t\right\} .
\end{aligned}
$$

Then $S:=S_{\Gamma} \cup S_{K}$ is a finite generating set for $K \backslash \Gamma$. Indeed, let $(f, \gamma) \in$ $K \backslash \Gamma$. For $x \in \mathrm{V}$, choose $\gamma_{x} \in \Gamma$ such that $\gamma_{x} o=x$ and write $h_{x} \in K_{*}^{\vee}$ for the function $h_{x}(o):=f(x)$ and $h_{x}(y):=\operatorname{id}_{K}$ for $y \neq o$. Then

$$
(f, \gamma)=\left(\prod_{\substack{x \in \mathrm{V} \\ f(x) \neq \mathrm{id}_{K}}}\left(\mathrm{id}_{\mathrm{V}}, \gamma_{x}\right)\left(h_{x}, \mathrm{id}_{\Gamma}\right)\left(\mathrm{id}_{\mathrm{V}}, \gamma_{x}^{-1}\right)\right)\left(\mathrm{id}_{\mathrm{V}}, \gamma\right),
$$

where the product over $x$ is taken in any order. If we then write each $\left(h_{x}, \mathrm{id}_{\Gamma}\right)$ as a product of $\left(F_{j}, \mathrm{id}_{\Gamma}\right)$, each $\left(\mathrm{id}_{\mathrm{V}}, \gamma_{x}\right)$ as a product of $\left(\mathrm{id}_{\mathrm{V}}, \gamma_{i}\right)$, each $\left(\operatorname{id}_{\mathrm{V}}, \gamma_{x}^{-1}\right)$ as a product of $\left(\mathrm{id}_{\mathrm{V}}, \gamma_{i}\right)$, and $\left(\mathrm{id}_{\mathrm{V}}, \gamma\right)$ as a product of $\left(\mathrm{id}_{\mathrm{V}}, \gamma_{i}\right)$, we obtain a representation as a product of elements of $S$.

The lamplighter groups $G_{d}$ are those where $\Gamma=\mathrm{V}=\mathbb{Z}^{d}$ and $K=\mathbb{Z}_{2}$. [By a theorem of Baumslag (1961), the groups $G_{d}$ are not finitely presentable.]

COROLLARY 6.8. Let $K$ be a finite group with more than one element. Let $\Gamma$ be a finitely generated group acting transitively on an infinite set $\mathrm{V}$. If $G$ is any Cayley graph of $K \backslash \Gamma$, then $p_{u}(G)<1$.

To prove this, we borrow a technique from Benjamini, Pemantle and Peres (1998): If $\phi$ and $\psi$ are two paths, denote by $|\phi \cap \psi|$ the number of edges they have in common (as sets of edges).

LeMma 6.9. Let $G$ be a graph and $x, y$ be any two vertices in $G$. Let $\theta \in(0,1)$ and $c>0$ be constants. Suppose that $\mu$ is a probability measure on ( possibly self-intersecting) paths $\psi$ joining $x$ to $y$ such that

$$
(\mu \times \mu)\{(\phi, \psi):|\phi \cap \psi|=n\} \leq c \theta^{n}
$$

for all $n \in \mathbb{N}$. Let $\theta<p<1$. Then for Bernoulli $(p)$ percolation on $G$, we have

$$
\tau(x, y) \geq c^{-1}(1-\theta / p) .
$$


Proof. Define the random variable

$$
Z:=\sum_{\psi} \mu(\psi) \frac{\mathbf{1}_{\{\psi \text { is open }\}}}{\mathbf{P}[\psi \text { is open }]} .
$$

Then $\mathbf{E}[Z]=1$ and

$$
\begin{aligned}
\mathbf{E}\left[Z^{2}\right] & =\sum_{\phi, \psi} \mu(\phi) \mu(\psi) \frac{\mathbf{P}[\phi, \psi \text { are both open }]}{\mathbf{P}[\phi \text { is open }] \mathbf{P}[\psi \text { is open }]} \\
& =\sum_{\phi, \psi} \mu(\phi) \mu(\psi) p^{-|\phi \cap \psi|} \\
& =\sum_{n} p^{-n} \sum_{|\phi \cap \psi|=n} \mu(\phi) \mu(\psi) \\
& \leq \sum_{n} p^{-n} c \theta^{n}=c(1-\theta / p)^{-1} .
\end{aligned}
$$

Therefore, the Cauchy-Schwarz inequality yields

$$
\tau(x, y) \geq \mathbf{P}[Z>0] \geq \mathbf{E}[Z]^{2} / \mathbf{E}\left[Z^{2}\right] \geq c^{-1}(1-\theta / p) .
$$

Proof of Corollary 6.8. Since $K$ is assumed to be finite, we take the generating set $\left\{k_{1}, \ldots, k_{t}\right\}$ to be all of $K$. We use the Cayley graph given by the generating set $S$. It suffices to exhibit a measure on paths connecting $\left(\mathrm{id}_{\mathrm{V}}, \mathrm{id}_{\Gamma}\right)$ to $(f, \gamma)$ that satisfies the condition of Lemma 6.9 with $\theta$ and $c$ not depending on $(f, \gamma)$, since then Theorem 4.1 implies that $p_{u} \leq \theta$.

Define edges joining pairs in $\mathrm{V}$ by

$$
\mathrm{E}:=\left\{\left[x, \gamma_{i}^{\varepsilon} x\right]: x \in \mathrm{V}, 1 \leq i \leq s, \varepsilon= \pm 1\right\}
$$

The graph $G^{\prime}:=(\mathrm{V}, E)$ will be called the base graph. Note that the base graph is not the same as the Cayley graph, $G=(\mathrm{V}(G), \mathrm{E}(G))$. Let $\pi: \mathrm{V}(G) \rightarrow \mathrm{V}$ be the projection $\pi(g, \beta):=\beta o$. Let $v_{1}, v_{2}, \ldots$ and $u_{1}, u_{2}, \ldots$ be infinite simple paths in $G^{\prime}$ starting at $v_{1}=o$ and $u_{1}=\gamma o$, respectively, that are disjoint, except that $v_{1}$ may equal $u_{1}$. Because $G^{\prime}$ is an infinite, connected, transitive graph, it is easy to show that such paths exist. For each $j=1,2, \ldots$, fix an $\alpha_{j} \in S_{\Gamma}$ such that $\alpha_{j} v_{j}=v_{j+1}$ and fix a $\beta_{j} \in S_{\Gamma}$ such that $\beta_{j} u_{j}=u_{j+1}$.

Given a word $W=w_{1} w_{2} \cdots w_{n}$ with letters from $S$, let $W^{-1}$ denote the word $w_{n}^{-1} w_{n-1}^{-1} \cdots w_{1}^{-1}$, and let $\tilde{W}(j), j \in\{0,1, \ldots, n\}$, denote the group element $w_{1} w_{2} \cdots w_{j}$.

Let $W_{\gamma}$ be a word in $S_{\Gamma}$ representing $\left(\mathrm{id}_{\mathrm{V}}, \gamma\right)$ with the property that for every $v \in \mathrm{V}$ such that $f(v) \neq \mathrm{id}_{K}$, there is a $j$ such that $\pi \tilde{W}(j)=v$. Let $n$ be the length of $W_{\gamma}$. Let $W_{\alpha}$ be the word $\alpha_{1} \alpha_{2} \cdots \alpha_{n}$, and let $W_{\beta}$ be the word $\beta_{1} \beta_{2} \cdots \beta_{n}$. Let $W$ be the concatenation

$$
W:=W_{\alpha} W_{\alpha}^{-1} W_{\gamma} W_{\beta} W_{\beta}^{-1} W_{\gamma}^{-1} W_{\alpha} W_{\alpha}^{-1} W_{\gamma} W_{\beta} W_{\beta}^{-1},
$$

and let $N$ be the length of $W$. Let the letters in $W$ be $W=w_{1} w_{2} \cdots w_{N}$. Consider words of the form $\phi(X):=w_{1} X_{1} w_{2} X_{2} \cdots w_{N} X_{N}$, where $X=$ 
$\left(X_{1}, \ldots, X_{N}\right) \in S_{K}^{N}=\left(S_{K}\right)^{N}$. For any $v \in \mathrm{V}$, let $J_{v}$ be the set of $j \in$ $\{1,2, \ldots, N\}$ such that $\pi \tilde{W}(j)=v$. Let $\pi_{K}: K \backslash \Gamma \rightarrow K_{*}^{\vee}$ be the projection onto the first coordinate. The uniform measure $\mu_{0}$ on the set of $X \in S_{K}^{N}$ such that $\phi(X)$ is a word representing $(f, \gamma)$ can be described as follows: $\prod_{j \in J_{v}} \pi_{K} X_{j}(o)=f(v)$ (where the order of multiplication is the order of $J_{v}$ as a subset of $\mathbb{N})$ and for every $j_{1} \in J_{v}$, the random variables $\left(X_{j}: j \in J_{v}, j \neq j_{1}\right)$ are independent, uniform in $S_{K}$ and independent of $\left(X_{j}: j \notin J_{v}\right)$. Note that $\phi(X)$ can also be thought of as a random path in $G$ from $\left(\operatorname{id}_{V}, \operatorname{id}_{\Gamma}\right)$ to $(f, \gamma)$.

Let $X$ and $Y$ be i.i.d. with law $\mu_{0}$. We want to bound the probability that there are $k$ edges shared by the paths $\phi(X)$ and $\phi(Y)$. In fact, we shall bound the probability that these paths share at least $k$ vertices. Given any $j \in\{1, \ldots, N\}$, let $H(j)$ be the set of $v \in \mathrm{V}$ such that $\min J_{v}<j<\max J_{v}$, and let $h(j):=|H(j)|$. The choice of $W$ ensures that

$$
h(j) \geq \min \{j, N-j, n\}-1 .
$$

Because $N=O(n)$, this gives

$$
h(j) \geq c_{1} \min \{j, N-j\}-1
$$

for some universal constant $c_{1}>0$. If $(g, \delta)$ is the element of $\hat{V}$ represented by the word $w_{1} X_{1} w_{2} \cdots w_{j} X_{j}$, then $(g(v): v \in H(j))$, are i.i.d. uniform in $K$. Consequently, the probability that $w_{1} X_{1} w_{2} \cdots w_{j} X_{j}=w_{1} Y_{1} w_{2} \cdots w_{j^{\prime}} Y_{j^{\prime}}$, as elements of $K \backslash \Gamma$, is at most $|K|^{-\max \left\{h(j), h\left(j^{\prime}\right)\right\}}$. If there are more than $8 k$ vertices common to $\phi(X)$ and $\phi(Y)$, then there must be a pair of indices $j, j^{\prime} \in\{k, k+1, \ldots, N-k\}$ such that $w_{1} X_{1} w_{2} \cdots w_{j} X_{j}=w_{1} Y_{1} w_{2} \cdots w_{j^{\prime}} Y_{j^{\prime}}$, as elements of $K \backslash \Gamma$, or with a similar equality when the rightmost letter is dropped from either or both sides. The probability for that is at most

$$
4 \sum_{j=k}^{N-k} \sum_{j^{\prime}=k}^{N-k}|K|^{-\max \left\{h(j), h\left(j^{\prime}\right)\right\}} \leq 4 \sum_{j=k}^{N-k} \sum_{j^{\prime}=k}^{N-k}|K|^{-\left(h(j)+h\left(j^{\prime}\right)\right) / 2} \leq c_{2}|K|^{-c_{1} k},
$$

where $c_{2}$ is a constant depending only on $|K|$. Consequently, as appeal to Lemma 6.9 completes the proof.

Benjamini and Schramm (1996) conjectured that for any nonamenable Cayley graph $G$, we have $p_{c}(G)<p_{u}(G)$. This is still open, but using our main result on cluster indistinguishability, we may show that a comparable statement fails for invariant percolation processes.

COROLLARY 6.10. There is an invariant deletion-tolerant percolation process $(\mathbf{P}, \omega)$ on a nonamenable Cayley graph that has only finite components a.s., but such that for every $\varepsilon>0$, the union of $\omega$ with an independent Bernoulli( $\varepsilon$ ) percolation process produces a unique infinite cluster a.s.

PRoOF. Recall that Bernoulli $(p)$ bond percolation on $\mathbb{Z}^{2}$ produces a.s. no infinite cluster iff $p \leq 1 / 2$ [see Grimmett (1989)]. Let $\mathbb{F}_{2}$ be the Cayley graph of the free group on two letters with the usual generating set. Let $G:=\mathbb{F}_{2} \times$ $\mathbb{Z}^{2}$. Each edge of $G$ joins vertices $\left(x_{1}, y_{1}\right)$ to $\left(x_{2}, y_{2}\right)$, whether either $x_{1}=x_{2}$ and $y_{1} \sim y_{2}$, or $x_{1} \sim x_{2}$ and $y_{1}=y_{2}$. The former type of edge will be called a 
$\mathbb{Z}^{2}$ edge, while the latter will be called an $\mathbb{F}_{2}$ edge. For each $x \in \mathbb{F}_{2}$, the set of all edges $\left[\left(x, y_{1}\right),\left(x, y_{2}\right)\right]$ is called the $\mathbb{Z}^{2}$ fiber on $x$. Let $\omega$ be Bernoulli(1/2) bond percolation on all the $\mathbb{Z}^{2}$ edges; it contains no $\mathbb{F}_{2}$ edge. Then $\omega$ is invariant and deletion tolerant. When we take the union with an independent $\operatorname{Bernoulli}(\varepsilon)$ process $\eta$, the intersection of $\omega \cup \eta$ with each $\mathbb{Z}^{2}$ fiber contains a.s. a unique infinite cluster. These clusters a.s. connect to each other in $G$. Thus, $\omega \cup \eta$ contains a unique infinite cluster whose intersection with each $\mathbb{Z}^{2}$ fiber has an infinite component. Since $\omega \cup \eta$ is insertion tolerant, cluster indistinguishability of $\omega \cup \eta$ implies that it has no other infinite cluster.

QUESTION 6.11. Is there an insertion-tolerant process with the property exhibited in Corollary 6.10?

The same line of thought is also used to prove the following theorem, which is an answer to a question posed by Yuval Peres (private communication) and motivated by the work of Salzano and Schonmann $(1997,1999)$ on contact processes.

TheOREM 6.12. Let $G, H$ and $H^{\prime}$ be unimodular transitive graphs. Assume that $H^{\prime} \subset H$ and $G$ is infinite. Then

$$
p_{u}\left(G \times H^{\prime}\right) \geq p_{u}(G \times H)
$$

In particular, when $H^{\prime}$ consists of a single vertex of $H$ we get

$$
p_{u}(G) \geq p_{u}(G \times H) \text {. }
$$

Proof. Let $\omega$ be $\operatorname{Bernoulli}(p)$ bond percolation on $G \times H$, where $p>$ $p_{u}\left(G \times H^{\prime}\right)$, and let $\Gamma$ be the product of a unimodular transitive automorphism group on $G$ with a unimodular transitive automorphism group on $H$. Then $\Gamma$ is a unimodular transitive automorphism group on $G \times H$.

Let $\hat{Z}:=\left\{\gamma\left(G \times H^{\prime}\right): \gamma \in \Gamma\right\}$. By Theorem 1.2 applied to $G \times H^{\prime}$, for every $Z \in \hat{Z}$ a.s. there is a unique infinite cluster, say $Q_{Z}$, of $\omega \cap Z$. Now let $Z, Z^{\prime} \in \hat{Z}$. We claim that a.s. $Q_{Z}$ and $Q_{Z^{\prime}}$ are in the same cluster of $\omega$. Indeed, since $G$ is infinite, there are infinite sequences of vertices $v_{j} \in Z, v_{j}^{\prime} \in Z^{\prime}$ such that $\operatorname{dist}\left(v_{j}, v_{j}^{\prime}\right)=\inf \left\{\operatorname{dist}\left(v, v^{\prime}\right): v \in Z, v^{\prime} \in Z^{\prime}\right\}$ for all $j$. Because $\inf _{j} \mathbf{P}\left[v_{j} \in Q_{Z}, v_{j}^{\prime} \in Q_{Z^{\prime}}\right]>0$, with positive probability $v_{j} \in Q_{Z}$ and $v_{j}^{\prime} \in Q_{Z^{\prime}}$ for infinitely many $j$, and by Kolmogorov's $0-1$ law this holds a.s. It is then clear that for some such $j$ there is a connection in $\omega$ between $v_{j}$ and $v_{j}^{\prime}$. It follows that a.s. there is a unique cluster $Q$ of $\omega$ that contains every $Q_{Z}, Z \in \hat{Z}$.

Let $\mathscr{A}$ be the set of all pairs $(C, W) \in 2^{\mathrm{V}(G \times H)} \times 2^{\mathrm{E}(G \times H)}$ such that $C$ meets an infinite component of $Z \cap W$ for every $Z \in \hat{Z}$. We know that $Q$ is the only cluster of $\omega$ with $(Q, \omega) \in \mathscr{A}$. By Theorem 3.3, it follows then that $Q$ is the only infinite cluster of $\omega$. 
REMARK 6.13. The same theorem holds without the assumption of unimodularity and with "transitive" replaced by "quasi-transitive." This follows similarly from the indistinguishability of robust properties proved by Häggström, Peres and Schonmann (1999).

REMARK 6.14. There are infinite Cayley graphs $H^{\prime} \subset H$ with $p_{u}\left(H^{\prime}\right)<$ $p_{u}(H)$. For example, one may take $H^{\prime}=\mathbb{Z}^{2}$ and let $H$ be the free product $\mathbb{Z}^{2} * \mathbb{Z}_{2}$, as in Remark 1.3.

The work of Schonmann (1999a) was motivated by an analogy between percolation and contact processes. More specifically, the property of having complete convergence with survival for a contact process is closely analogous to having uniqueness of the infinite cluster for percolation. However, Remark 6.14 describes an instance where this analogy fails, because complete convergence with survival is a property which is monotone in the graph [Salzano and Schonmann (1997)].

Acknowledgments. We are grateful to Itai Benjamini, Olle Häggström, Yuval Peres and Roberto Schonmann for fruitful conversations and helpful advice.

\section{REFERENCES}

Aizenman, M., Kesten, H. and Newman, C. M. (1987). Uniqueness of the infinite cluster and continuity of connectivity functions for short- and long-range percolation. Comm. Math. Phys. 111 505-532.

Aizenman, M. and Newman, C. M. (1984). Tree graph inequalities and critical behavior in percolation models. J. Statist. Phys. 36 107-143.

AleXANDER, K. (1995). Simultaneous uniqueness of infinite clusters in stationary random labeled graphs. Comm. Math. Phys. 168 39-55.

BABSon, E. and Benjamini, I. (1999). Cut sets and normed cohomology with applications to percolation. Proc. Amer. Math. Soc. 127 589-597.

BAUMSLAG, G. (1961). Wreath products and finitely presented groups. Math. Z. 75 22-28.

Benjamini, I., Lyons, R., Peres, Y. and Schramm, O. (1999). Group-invariant percolation on graphs. Geom. Funct. Anal. 9 29-66.

Benjamini, I., Lyons, R. and Schramm, O. (1999). Percolation perturbations in potential theory and random walks. In Random Walks and Discrete Potential Theory (H. Picardello and W. Woess, eds.) 56-84. Cambridge Univ. Press.

Benjamini, I., Pemantle, R. and Peres, Y. (1998). Unpredictable paths and percolation. Ann. Probab. 26 1198-1211.

Benjamini, I. and Schramm, O. (1996). Percolation beyond $\mathbb{Z}^{d}$, many questions and a few answers. Electronic Comm. Probab. 1 71-82.

Benjamini, I. and Schramm, O. (1998). Percolation in the hyperbolic plane. Unpublished manuscript.

Burton, R. M. and Keane, M. (1989). Density and uniqueness in percolation. Comm. Math. Phys. 121 501-505.

DE LA HaRPe, P. and VAlette, A. (1989). La propriété $(T)$ de Kazhdan pour les groupes localement compacts (avec un appendice de Marc Burger). Astérisque 175.

Delorme, P. (1977). 1-cohomologie des représentations unitaires des groupes de Lie semisimples et résolubles. Produits tensoriels continus et représentations. Bull. Soc. Math. France 105 281-336. 
Gandolfi, A., Keane, M. S. and Newman, C. M. (1992). Uniqueness of the infinite component in a random graph with applications to percolation and spin glasses. Probab. Theory Related Fields 92 511-527.

Glasner, E. and WEISs, B. (1997). Kazhdan's property $T$ and the geometry of the collection of invariant measures. Geom. Funct. Anal. 7 917-935.

Grimmett, G. R. (1989). Percolation. Springer, New York.

Grimmett, G. R. and Newman, C. M. (1990). Percolation in $\infty+1$ dimensions. In Disorder in Physical Systems (G. R. Grimmett and D. J. A. Welsh, eds.) 167-190. Clarendon Press, Oxford.

Guichardet, A. (1977). Étude de la 1-cohomologie et de la topologie du dual pour les groupes de Lie à radical abélien. Math. Ann. 228 215-232.

HÄGGSTRÖM, O. (1997). Infinite clusters in dependent automorphism invariant percolation on trees. Ann. Probab. 25 1423-1436.

HäGgströM, O. and Peres, Y. (1999). Monotonicity of uniqueness for percolation on Cayley graphs: all infinite clusters are born simultaneously. Probab. Theory Related Fields $113273-285$.

HägGström, O., Peres, Y. and Schonmann, R. H. (1999). Percolation on transitive graphs as a coalescent process: relentless merging followed by simultaneous uniqueness. In Perplexing Probability Problems: Papers in Honor of H. Kesten (M. Bramson and R. Durrett, eds.) 69-90. Birkhäuser, Boston.

Kaimanovich, V. A. and Vershik, A. M. (1983). Random walks on discrete groups: boundary and entropy. Ann. Probab. 11 457-490.

Lalley, S. P. (1998). Percolation on Fuchsian groups. Ann. Inst. H. Poincaré Probab. Statist. 34 $151-177$.

Liggett, T. M., Schonmann, R. H. and Stacey, A. M. (1997). Domination by product measures. Ann. Probab. 25 71-95.

Lyons, R. (1990). Random walks and percolation on trees. Ann. Probab. 18 931-958.

Lyons, R., Pemantle, R. and Peres, Y. (1996). Random walks on the Lamplighter Group. Ann. Probab. 24 1993-2006.

Lyons, R. and Peres, Y. (1998). Probability on Trees and Networks. Cambridge Univ. Press. To appear. Available at http://php.indiana.edu/ rdlyons $/$.

LyONs, R. and Schramm, O. (1999). Stationary measures for random walks in a random environment with random scenery. New York J. Math. 5 107-113.

Newman, C. M. and Schulman, L. S. (1981). Infinite clusters in percolation models. J. Statist. Phys. 26 613-628.

Peres, Y. (1999). Percolation on nonamenable products at the uniqueness threshold. Ann. Inst. J. Poincaré Probab. Statist. To appear.

Salzano, M. and Schonmann, R. H. (1997). The second lowest extremal invariant measure of the contact process. Ann. Probab. 25 1846-1871.

Salzano, M. and Schonmann, R. H. (1999). The second lowest extremal invariant measure of the contact process II. Ann. Probab. 27 845-875.

Schonmann, R. H. (1999a). Stability of infinite clusters in supercritical percolation. Probab. Theory Related Fields 113 287-300.

Schonmann, R. H. (1999b). Percolation in $\infty+1$ dimensions at the uniqueness threshold. In Perplexing Probability Problems: Papers in Honor of H. Kesten (M. Bramson and R. Durrett, eds.) 53-67. Birkhäuser, Boston.

Trofimov, V. I. (1985). Groups of automorphisms of graphs as topological groups. Math. Notes $38717-720$.

ZuK, A. (1996). La propriété (T) de Kazhdan pour les groupes agissant sur les polyèdres. C. $R$. Acad. Sci. Paris Sér. I Math. 323 453-458.

Department of Mathematics

INDIANA UNIVERSITY

BloOMington, Indiana 47405-5701

E-MAIL: rdlyons@indiana.edu
Department of Mathematics

WEIZMANn Institute of ScIEnce

REHOVOT 76100

ISRAEL

E-MAIL: schramm@wisdom.weizmann.ac.il 